\title{
ASYMMETRIC ROTATOR MODEL OF ODD-MASS NUGLEI
}

\author{
KARL T. HECHT \\ Randall Laboratory of Physics, University of Michigan, Ann Arbor, Michigan ${ }^{\dagger}$ \\ and \\ G. R. SATCHLER \\ Oak Ridge National Laboratory, Oak Ridge, Tennessee
}

Received 25 September 1961

\begin{abstract}
The asymmetric rotator model of Davydov and Filippov has been extended to oddmass nuclei by coupling a single nucleon to an inert core of well stabilized asymmetric equilibrium shape. Rotational energies are calculated for states with spin $I$ through numerical diagonalization of $\left(I+\frac{1}{2}\right) \times\left(I+\frac{1}{2}\right)$ rotational matrices which depend in a complicated way on the state of the odd nucleon. The state of the odd nucleon is described by single particle wave functions such as those calculated by Newton, generalizations for the asymmetric case of the wave functions computed by Nilsson for axially symmetric nuclei. The rotational energy spectrum for a given particle excitation is in general very rich in number of levels and may consist of a complicated sequence of spin values. In many cases, however, particularly for small asymmetries, the rotational spectra may consist of several well separated or overlapping sequences of spin states which resemble the rotational bands of axially symmetric nuclei, especially insofar as $K$ (which gives the projection of $I$ on the body-fixed $z$-axis) may be approximately a good quantum number for each sequence.

In an initial survey of odd-mass nuclei around $A=190$, no clear-cut evidence has been found for the existence of nuclei with a well defined asymmetric equilibrium shape. Calculations for $I^{191}$ and $R^{185}$ indicate only that it may be very difficult to distinguish between a symmetric and an asymmetric rotator model when the asymmetry is small. Calculations for $\mathrm{Pt}^{195}$ show that, aithough the observed level scheme can be reproduced by asymmetric rotator theory, the observed electromagnetic transition probabilities are not in agreement with the predictions of the simple asymmetric rotator model.
\end{abstract}

\section{Introduction}

Since Davydov and Filippov ${ }^{1}$ ) first suggested the validity of the asymmetric rotator modification for the strong coupling limit of Bohr's collective model ${ }^{2}$ ), surveys have been made by several authors ${ }^{3}$ ) which seem to indicate that the model, in which the nucleus is pictured to have a well stabilized axially asymmetric equilibrium shape, may successfully describe the properties of low-lying levels of even nuclei in many different regions of the periodic table. However, since the $I=2$ and 3 rotator levels particularly in the limiting case of large asymmetry, have properties very similar to those predicted on the basis of the vibrational model, the validity of the asymmetric rotator model has been

t This work was supported in part by the Office of Naval Research under Navy Theoretical Physics Contract No. Nonr 1224(15). 
questioned by other authors who point out that many of the predictions of the Davydov-Filippov model are also in quantitative agreement with the vibrational model with appropriate refinements ${ }^{4}$ ). The calculations of Newton ${ }^{5}$ ), on the other hand, indicate that the axially symmetric equilibrium shape of the Nilsson model nucleus ${ }^{6}$ ) is perhaps not always the intrinsically most stable. It was therefore thought worthwhile to extend the asymmetric rotator model to the case of odd-mass nuclei. In order to afford a further possible test of the model, however, the nuclei to be studied should have preferably both large asymmetry and, equally important, a well defined equilibrium shape, that is small vibrationrotation interactions. The work of Davydov ${ }^{7}$ ) on even nuclei suggests that the sequence $\mathrm{Os}^{186}, \mathrm{Os}^{188}, \mathrm{Os}^{190}$ and $\mathrm{Os}^{192}$ may satisfy both these requirements better than nuclei in other regions of the periodic table. The theory will therefore be applied in particular to odd-mass nuclei in the vicinity of $A=\mathbf{1 9 0}$.

\section{The Model}

It will be assumed that a single nucleon is coupled to an inert core of asymmetric equilibrium shape. The rotational Hamiltonian ${ }^{2}$ ) has the form

$$
H_{\text {rot }}=\frac{\hbar^{2}}{2 \mathscr{I}_{x}}\left(I_{x}-j_{x}\right)^{2}+\frac{\hbar^{2}}{2 \mathscr{I}_{y}}\left(I_{y}-j_{y}\right)^{2}+\frac{\hbar^{2}}{2 \mathscr{I}_{z}}\left(I_{z}-j_{z}\right)^{2}
$$

where the inertial parameters are such that $\mathscr{I}_{x} \neq \mathscr{I}_{y} \neq \mathscr{I}_{z}$. The operators $I_{\boldsymbol{k}}$ and $j_{k}$ describe the body fixed components of the total angular momentum of the nucleus and the angular momentum of the odd nucleon, respectively. For the present it will be assumed that the wave function which describes the motion of the odd nucleon in the ellipsoidal potential field is known in the form

$$
\chi_{\mathrm{partclel}}=\sum_{j \Omega} c_{j \Omega} \chi_{\Omega^{j}} \equiv \chi_{\nu},
$$

where $\chi_{\Omega}^{j}$ are eigenfunctions of $j^{2}$ and $j_{z}$. In the limit of zero deformation (spherical shell model limit) all but one of the coefficients $c_{j \Omega}$ are equal to zero in a particular state. In the limit of axial symmetry $\Omega$ is a good quantum number, and the summation extends only over the possible values of $j$. In the asymmetric case ellipsoidal symmetry still imposes some restrictions. The Hamiltonian can depend on the angular coordinates of the odd nucleon, defined relative to the principal axis system of the ellipsoid, only through the spherical harmonics, $Y_{0}{ }^{2}, Y_{2}^{2}$ and $Y_{-2}^{2}$. As a result the summation over $\Omega$ will involve either the values $\left(+\frac{1}{2} \pm 2 n\right)$ or $\left(-\frac{1}{2} \pm 2 n\right)$ with $n=0,1,2, \ldots$ The two types of coefficients are related by $c_{j-\Omega}=(-1)^{j-\frac{1}{2}} c_{j \Omega}$. The complementary state $\chi_{-\nu}$, formed with $\sum_{j \Omega} c_{j-\Omega} \chi_{-\Omega}^{j}$, is degenerate with $\chi_{\nu}$ as in the axially symmetric limit. In the strong coupling approximation the wave function of the system is made up of products of the particle wave functions and the 
rotational wave functions $D_{M K}^{I}$. (The wave function which describes the zero point vibrational motion will be understood but not explicitly written.) The total wave function must have the specific symmetry ${ }^{2}$ )

$$
\begin{aligned}
\psi=\sum_{K}^{\prime} C_{K} \psi_{K}=\sqrt{\frac{2 I+1}{16 \pi^{2}} \sum_{K}^{\prime} C_{K} \sum_{j \Omega}^{\prime} c_{j \Omega}\left\{D_{M K}^{I} \chi_{\Omega}^{j}+(-1)^{I-j} D_{M-K}^{I} \chi_{-\Omega}^{j}\right\}} \\
=\sqrt{\frac{2 I+1}{16 \pi^{2}}} \sum_{K}^{\prime} C_{K}\left\{D_{M K}^{I} \chi_{\nu}+(-1)^{I-1} D_{M-K}^{I} \chi_{-\nu}\right\},
\end{aligned}
$$

where $(K-\Omega)$ must be an even integer. This can be accomplished by restricting the summation over both $K$ and $\Omega$ to the set of values

$$
\ldots,-\frac{11}{2},-\frac{7}{2},-\frac{3}{2}, \frac{1}{2}, \frac{5}{2}, \frac{9}{2}, \frac{13}{2}, \ldots
$$

This restriction is indicated by the prime on the summation symbols. The coefficients $C_{K}$ which determine the $K$-admixtures in a given rotational state are to be determined from the solution of the rotational problem, unlike the coefficients $c_{j \Omega}$ which are assumed known for each state of particle excitation. In the axially symmetric limit $K$ is a good quantum and all but one of the coefficients, $C_{K}$, go to zero. Similarly only one $\Omega$ remains, although there is still a sum over $j$. We then have $K=\Omega$, (the $K \neq \Omega$ states are infinitely high in energy). The above convention for the values assumed by $K$ and $\Omega$ means that the wave function reduces to that of Bohr and Mottelson ${ }^{2,6}$ ) for $K=\Omega=$ $\left(\frac{1}{2}+2 n\right)$ in the symmetric limit, but to this function multiplied by $(-1)^{I-j}$ for $K=\Omega=\left(-\frac{1}{2}+2 n\right)$, where $n$ is integral.

\section{Rotational Energies}

It will be useful to introduce the rotational angular momentum operator $\mathbf{R},(\mathbf{R}=\mathbf{I}-\mathbf{j})$. In terms of $\mathbf{R}$, the rotational Hamiltonian has the simple form

$$
H_{\text {rot }}=\frac{\hbar^{2}}{2 \mathscr{I}_{x}} R_{x}{ }^{2}+\frac{\hbar^{2}}{2 \mathscr{I}_{y}} R_{y}{ }^{2}+\frac{\hbar^{2}}{2 \mathscr{I}_{z}} R_{z}{ }^{2}
$$

which will be particularly useful if a change is made from the $|I K j \Omega\rangle$ representation of eq. (3) to an $\left|I j R K_{R}\right\rangle$ representation ( $R$ and $K_{R}$ are the quantum numbers which determine the magnitude and the body-fixed $z$-component of the rotational angular momentum, respectively):

$$
\sqrt{\frac{\overline{2 I+1}}{8 \pi^{2}}} D_{M K}^{I} \chi_{\Omega^{j}}=\sum_{R}(-1)^{j-s_{\Delta}}\left\langle I j K-\Omega \mid I j R K_{R}\right\rangle \psi_{K_{R}}^{I j R} .
$$

The unusual phase factor and the unusual sign in the Clebsch-Gordan coefficient arise from the fact that the angular momentum coupled wave 
function is constructed for the case of angular momentum subtraction rather than addition $\left(\mathbf{R}=\mathbf{I}-\mathbf{j} ; K=K_{R}-\Omega\right)^{\dagger}$.

Davydov ${ }^{8}$ ) has treated the special case of an odd-mass nucleus in which the intrinsic particle state is assumed to be such that $j$ is a good quantum number and has the special value $j=\frac{1}{2}$. The $\left|I j R K_{R}\right\rangle$ representation is particularly useful to show that the rotational energies in this very special case have exactly the same values as the rotational energies of even nuclei with corresponding values of $R$. Each rotational energy level, however, is doubly degenerate corresponding to the two possible values $I=R \pm \frac{1}{2}$. The total wave function, of symmetry given by eq. (3), leads to a rotational wave function made up of terms of the form

$$
\frac{1}{\sqrt{ } 2}\left(\psi_{K_{R}}^{I j R}+(-1)^{R} \psi_{-K_{R}}^{I j R}\right) \quad\left(K_{R} \text { even }\right)
$$

In the special case $R=3$, for example, only one such linear combination exists, corresponding to $\left|K_{R}\right|=2$. When the quantum numbers have the values $R=3,\left|K_{R}\right|=2, j=\frac{1}{2}$, the total spin $I$ can have the two possible values $\frac{5}{2}$ and $\frac{7}{2}$, so that two independent (orthogonal) wave functions can be constructed with $R=3$. Application of eq. (5) gives with $I=\frac{5}{2}$

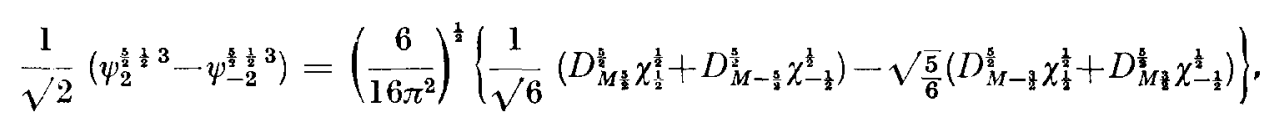

and with $I=\frac{7}{2}$

$$
\frac{1}{\sqrt{ } 2}\left(\psi_{2}^{\frac{2}{2} \frac{1}{2}}-\psi_{-2}^{\frac{7}{2} \frac{1}{2}}\right)=\left(\frac{8}{16 \pi^{2}}\right)^{\frac{2}{3}}\left\{\frac{\sqrt{ } 3}{2}\left(D_{M \frac{1}{2}}^{2} \chi_{\frac{1}{2}}^{\frac{1}{2}}-D_{M-\frac{1}{2}}^{\frac{2}{2}} \chi_{-\frac{1}{2}}^{\frac{1}{2}}\right)-\frac{1}{2}\left(D_{M-\frac{1}{2}}^{\frac{2}{2}} \chi_{\frac{1}{2}}^{\frac{1}{2}}-D_{M \frac{2}{2}}^{2} \chi_{-\frac{1}{2}}^{\frac{1}{2}}\right)\right\} .
$$

Since both these wave functions result in exactly the same linear combination of $R$ and $K_{R}$ values, and since the matrix elements of the rotational Hamiltonian can be functions of the quantum numbers $R$ and $K_{R}$ only, (as can be seen from eq. (4)), both these states have the same rotational energy, Also, since the rotational Hamiltonian operates on an eigenfunction of $\mathbf{R}$ in exactly the same way in which the rotational Hamiltonian for an even nucleus operates on an eigenfunction of $\mathbf{I}$, this energy has the same value as the rotational energy of an even nucleus with $I=3$. Altogether there are three independent wave functions with $I=\frac{5}{2}$ and four with $I=\frac{7}{2}$, corresponding to the possible number of values of $|K|$. The two $I=\frac{5}{2}$ states orthogonal to the $R=3$ state above have asymmetric rotator wave functions with $R=2$ and energies which are identical with those of the two possible $I=\frac{3}{2}$ states. The complete energy spectrum consists of a nondegenerate $I=\frac{1}{2}(R=0)$ ground state, the two different doubly degenerate $I=\frac{3}{2}, \frac{5}{2}(R=2)$ states, the single doubly de-

† A similar representation has been used by Osborn and Klema, Nuovo Cimento 9 (1958) 791. 
generate $I=\frac{\mathbf{5}}{\mathbf{2}}, \frac{\mathbf{7}}{\mathbf{2}}(R=3)$ state, three different doubly degenerate $I=\frac{7}{\mathbf{2}}$, $\frac{9}{2}(R=4)$ states, and so forth, all with the same energies as the analogous rotational states in even nuclei ${ }^{1}$ ). If the particle wave function is only approximately a pure $j=\frac{1}{2}$ function, the degeneracies are removed and the rotational energy spectrum should consist of doublets with $I=R \pm \frac{1}{2}$. Since no example is known for which $j=\frac{1}{2}$ is even a moderately good approximation this very special case is of academic interest only. However, it does illustrate a general feature. The rotational energy spectrum of an asymmetric odd-mass nucleus may be very rich in total number of levels compared with an axially symmetric nucleus.

In the strong coupling approximation the particle wave function in a given state is well specified independently of the rotational state of the nucleus. In an odd-mass asymmetric nucleus a realistic particle wave function in general involves many different values of $j$ and $\Omega$, so that products of the form $\left(\sum_{j \Omega} c_{j \Omega} \chi_{\Omega}{ }^{j}\right) D_{M K}^{I}$, lead to rotational wave functions which are linear combinations of many different values of $R$. Since $R$ is no longer a good quantum number, there will be no simple correlation between the rotational states of odd-mass and even nuclei, and the rotational energies will depend in a complicted way on the state of the odd nucleon. Either the $|I K j \Omega\rangle$ or the $\left|I j R K_{R}\right\rangle$ representation can be used to compute the matrix elements of the rotational Hamiltonian. For given $I$ (and specified particle excitation) there will be $\left(I+\frac{1}{2}\right)$ independent wave functions corresponding to the $\left(I+\frac{1}{2}\right)$ possible values of $|K|$, so that the determination of the rotational energies with spin $I$ will involve the diagonalization of $\left(I+\frac{1}{2}\right) \times\left(I+\frac{1}{2}\right)$ matrices, (provided the strong coupling limit applies; that is, provided the rotational energies are small compared with the single particle excitations so that matrix elements of the rotational Hamiltonian between different particle states can be neglected). In terms of the rotational constants.

$$
A_{1}=\hbar^{2} / 2 \mathscr{I}_{x}, \quad A_{2}=\hbar^{2} / 2 \mathscr{I}_{y}, \quad A_{3}=\hbar^{2} / 2 \mathscr{I}_{z},
$$

the rotational energy for the state $I=\frac{1}{2},\left(K=\frac{1}{2}\right)$ is given by

$$
\begin{aligned}
\left(I=\frac{1}{2}\right): \quad E_{\text {rot }}=-\frac{1}{2}\left(A_{1}+A_{2}\right)(a+b)-\frac{1}{2}\left(A_{1}-A_{2}\right) c+A_{3}\left\langle K_{R}{ }^{2}\right\rangle \\
\\
+\left(A_{1}+A_{2}\right) d+\left(A_{1}-A_{2}\right) e,
\end{aligned}
$$

where the parameters $a, b, c, d$ and $e$, and $\left\langle K_{R}{ }^{2}\right\rangle$ are determined by the state of the odd nucleon. The parameter $a$ is the decoupling parameter familiar from the theory of axially symmetric nuclei

$$
a==\sum_{j} c_{j \frac{1}{2}}^{2}(-1)^{j-\frac{1}{2}}\left(j+\frac{1}{2}\right) .
$$

In the asymmetric case it is replaced by

$$
a+b=\sum_{j \Omega}^{\prime} c_{j \Omega} c_{j,-(\Omega-1)}(-1)^{j-\frac{1}{2}}[(j+\Omega)(j-\Omega+1)]^{\frac{1}{2}} .
$$


The parameter $c$ involves a similar sum

while

$$
c=\sum_{j \Omega \mid} c_{j \Omega} c_{j .-(\Omega+1)}(-1)^{j-1}[(j-\Omega)(j+\Omega+1)]^{\mathbf{1}},
$$

$$
\left\langle K_{R}^{2}\right\rangle=\sum_{j \Omega}^{\prime} c_{j \Omega}^{2}(K-\Omega)^{2},
$$

with $K=\frac{1}{2}$ for $I=\frac{1}{2}$. The term $\left(A_{1}+A_{2}\right) d+\left(A_{1}-A_{2}\right) e$ has been purposely separated from the main terms in the rotational energy. It has the value

$$
\begin{aligned}
\frac{1}{2}\left(A_{1}+A_{2}\right) \sum_{j \Omega}^{\prime} c_{j \Omega}^{2}\left[j(j+1)-\Omega^{2}\right. & \left.+\frac{1}{2}\right]+\frac{1}{2}\left(A_{1}-A_{2}\right) \sum_{j \Omega}^{\prime} c_{j \Omega} c_{j(\Omega-2)} \\
& \times[(j+\Omega)(j-\Omega+1)(j+\Omega-1)(j-\Omega+2)]^{t}
\end{aligned}
$$

and is an energy contribution common to all states $I$. (It arises from the term $A_{1} j_{x}{ }^{2}+A_{2} j_{y}{ }^{2}$ in the rotational Hamiltonian, and could in principle be lumped together with the particle energy. In all numerical computations, however, it will be included in the rotational energy.) For $I>\frac{1}{2}$, matrix elements of the rotational Hamiltonian have been computed between the different possible states $\psi_{K}$ of eq. (3). They are shown explicitly in table 1 for $I=\frac{3}{2}$ to $\frac{13}{2}$ in terms of the rotational constants, the particle parameters $a, b$ and $c$, and the different values of $\left\langle K_{R}^{2}\right\rangle$. The common term $\left(A_{1}+A_{2}\right) d+\left(A_{1}-A_{2}\right) e$ must be added to the rotational energies computed from these matrix elements.

Diagonalization of the $\left(I+\frac{1}{2}\right) \times\left(I+\frac{1}{2}\right)$ matrices of table 1 will yield reliable rotational energies only for particle states which are well separated from other particle excitations since the rotational Hamiltonian, eq. (1), can couple different particle states. In the special case in which two different particle states lie close together the two sets of coupled rotational energies can be obtained from the diagonalization of $(2 I+1) \times(2 I+1)$ rotational matrices built up from the two coupled $\left(I+\frac{\mathbf{1}}{\mathbf{2}}\right) \times\left(I+\frac{1}{2}\right)$ rotational matrices for the different states. Matrix elements diagonal in the particle wave function have the form of table 1 , with particle parameters, $a, b$ etc. defined for each state, and with the two different particle energies added to the diagonal matrix elements. Matrix elements off-diagonal in the particle wave function again have the form of the matrix elements of table 1 provided that products of the form $c_{j: 3} c_{j \Omega^{\prime}}$ in the parameters $a, b, c, d, e$ and $\left\langle K_{R}{ }^{2}\right\rangle$ are replaced by $c_{j \Omega}^{(\mathbf{1})} c_{j \Omega^{\prime}}^{(2)}$ where the superscripts refer to the two different particle states. Terms not proportional to $a, b$, etc. must be omitted in matrix elements off-diagonal in the particle states. They are zero through the orthogonality of the different particle wave functions $\Sigma_{j \Omega} c_{j \Omega}^{(1)} c_{j \Omega}^{(2)}=0$.

\section{The Particle Wave Functions}

So far no specific assumptions have been made about the rotational constants $\left(A_{k}=\hbar^{2} / 2 \mathscr{I}_{k}\right)$ or the particle wave functions describing the odd nucleon. In 

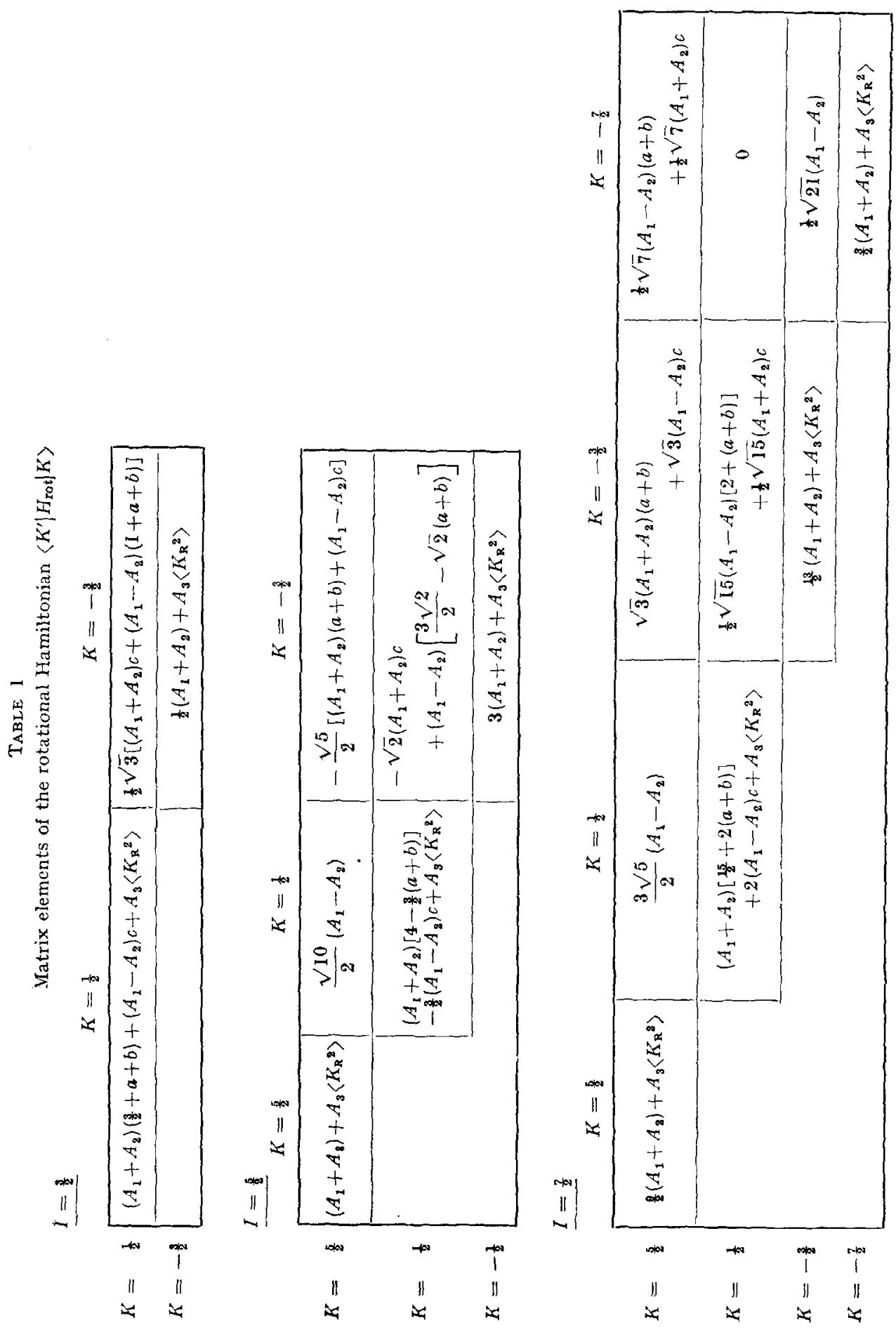

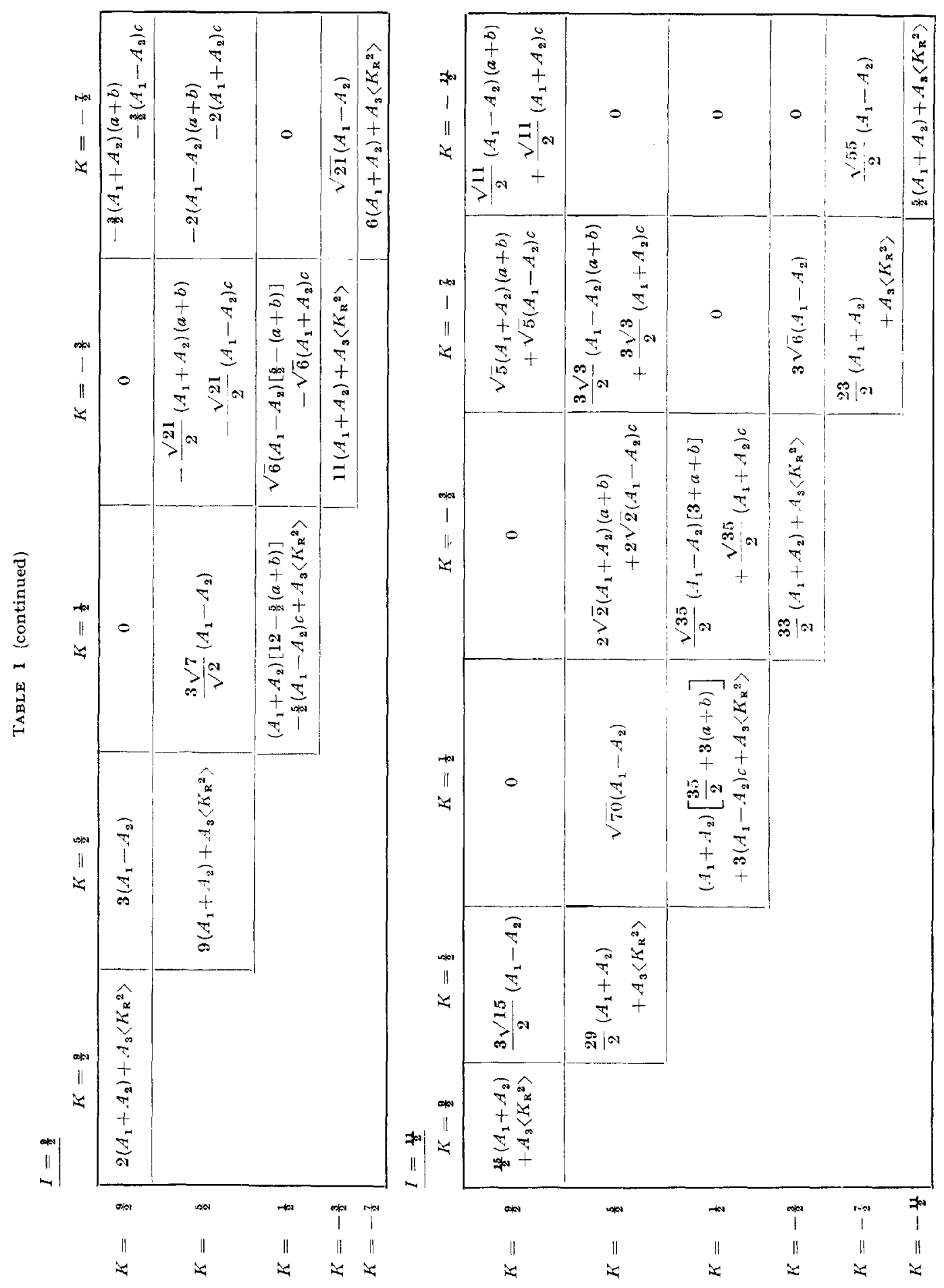


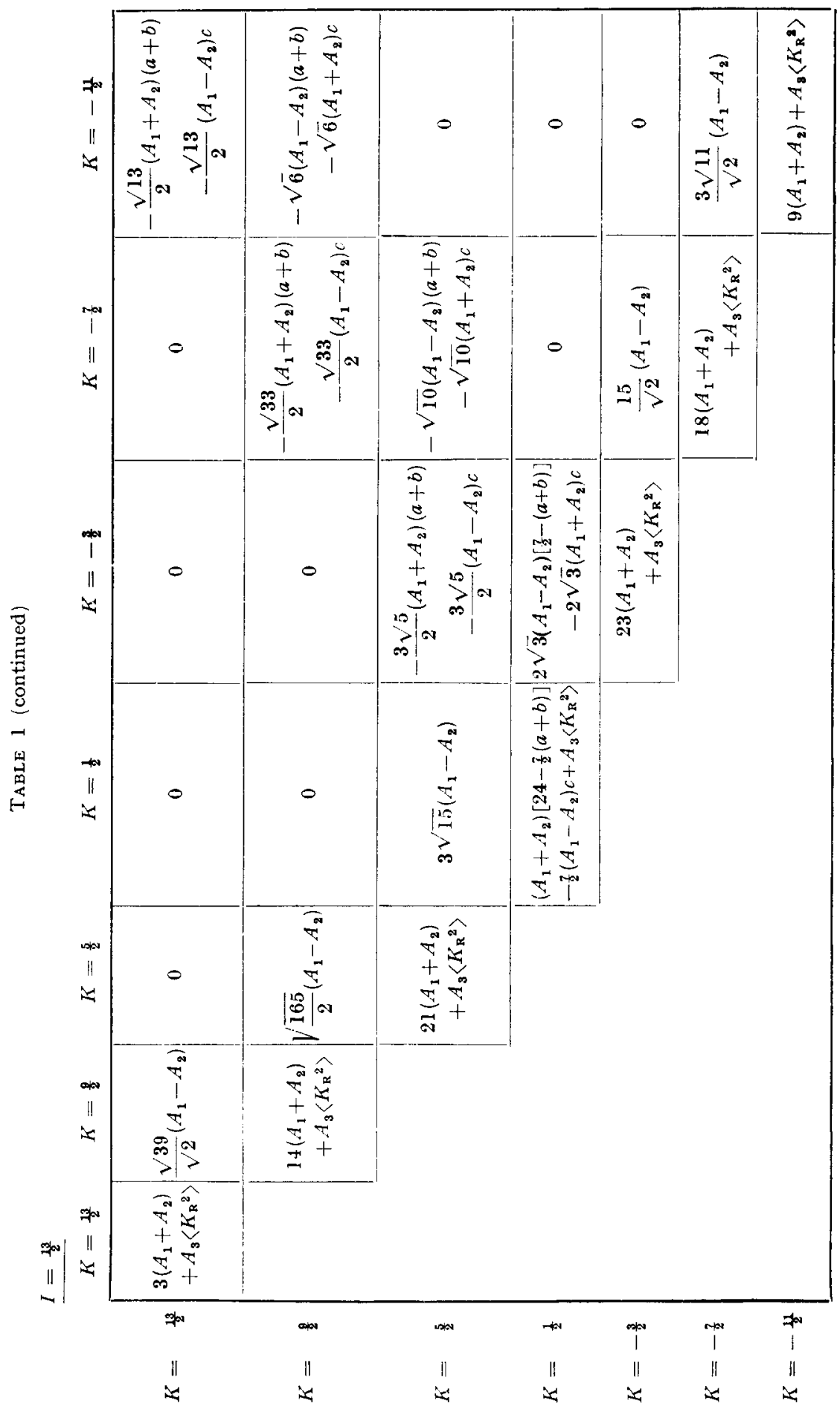


subsequent calculations the inertial parameters suggested by Bohr's hydrodynamical model $^{2}$ ) will be used

$$
\mathscr{I}_{k}=4 B \beta^{2} \sin ^{2}\left(\gamma-\frac{2}{3} \pi k\right) \quad(k=1,2,3=x, y, z),
$$

where, however, $B$ is to be determined empirically. The state of the odd nucleon will be described by wave functions such as those calculated by Newton ${ }^{5}$ ), that is by the eigenfunctions of a particle in an anisotropic oscillator potential with Nilsson spin-orbit coupling and $\mathbf{1}^{2}$ term. The single particle Hamiltonian is

$$
H_{\mathrm{partlcle}}=-\frac{\hbar^{2}}{2 m} \nabla^{2}+\frac{1}{2} m\left(\omega_{x} x^{2}+\omega_{y} y^{2}+\omega_{z} z^{2}\right)+C \mathbf{1} \cdot \mathbf{s}+D \mathbf{1}^{2},
$$

with

$$
\begin{gathered}
\omega_{k}=\omega_{0}(\beta, \gamma)\left[1+\sqrt{5 / 4 \pi} \beta \cos \left(\gamma-\frac{2}{3} \pi k\right)\right]^{-1} \quad(k=1,2,3=x, y, z), \\
\omega_{x} \omega_{y} \omega_{z}=\left[\omega_{0}(\beta=\gamma=0)\right]^{3} .
\end{gathered}
$$

In the notation of Nilsson ${ }^{6}$ ) we have

$$
C=-2 \kappa \hbar \omega_{0}, \quad D=-\kappa \mu \hbar \omega_{0} .
$$

As pointed out by Nilsson, the eigenfunctions of the Hamiltonian (15) can be treated in two possible ways. The Hamiltonian can be diagonalized by neglecting matrix elements off-diagonal in the principal harmonic oscillator quantum number $N$. In this case the particle eigenfunctions are expanded naturally in terms of angular momentum eigenfunctions in real physical space, for example in the $\left|N l \frac{1}{2} j \Omega\right\rangle$ representation. Alternately, by introducing the change of variables

$$
x=\left(\hbar / m \omega_{x}\right)^{\frac{1}{2} \xi}, \quad y=\left(\hbar / m \omega_{y}\right)^{\frac{1}{2}} \eta, \quad z=\left(\hbar / m \omega_{z}\right)^{\frac{1}{2} \zeta},
$$

the single particle Hamiltonian can be made rigorously diagonal in $N$ if the orbital angular momentum operator $\mathbf{1}$ for the real physical space is replaced by the infinitesimal rotation operator $\mathbf{l}_{\mathfrak{t}}$ in the pseudo-space $\xi, \eta, \zeta$, leading to the Hamiltonian

$$
H=\frac{1}{2} \hbar \omega_{x}\left(-\nabla_{\xi}^{2}+\xi^{2}\right)+\frac{1}{n} \hbar \omega_{n}\left(-\nabla_{n}^{2}+n^{2}\right)+\frac{1}{n} \hbar \omega_{r}\left(-\nabla_{\underline{\underline{z}}}{ }^{2}+\zeta^{2}\right)+C \mathbf{l}_{+} \cdot \mathbf{s}+D \mathbf{l}_{\mathrm{t}}{ }^{2} .
$$

Diagonalization of the Hamiltonian (19) leads to particle eigentunctions which are expanded in terms of angular momentum eigenfunctions in the pseudospace $\xi, \eta, \zeta$, that is in an $\left|N l_{t} \frac{1}{2} j_{t} \Omega_{t}\right\rangle$ representation. Newton has chosen to diagonalize the Hamiltonian in the form of eq. (19). For the present work Newton's calculations have been extended to include the $N=\mathbf{5}$ and $\mathbf{6}$ harmonic oscillator states and $N=\mathbf{4}$ states with parameters appropriate for odd-proton 
nuclei ${ }^{\dagger}$. (See figs. 1 and 2 in this paper and Mottelson and Nilsson ${ }^{9}$ )). The particle wave functions are therefore known in the form of eq. (2), but as linear combinations of angular momentum eigenfunctions in the pseudo-space $\xi, \eta, \zeta$. The formulae for the rotational energies, on the other hand, as well as all subsequent formulae for electromagnetic moments and transition probabilities, particle widths, etc. are given in terms of expansion coefficients involving the angular momentum eigenfunctions in real physical space. To first order in $\sqrt{5 / 4 \pi} \beta$, however, the two types of functions are identical. Since the model can be expected to have approximate validity at best, no distinction will be made between the two types of eigenfunctions.

If the distinction were to be taken literally, the operator $j_{x}$ in the rotational Hamiltonian, for example, could be written

$j_{x}=s_{x}+l_{x}=s_{x}+\frac{1}{2}\left(\sqrt{\frac{\omega_{z}}{\omega_{y}}}-\sqrt{\frac{\omega_{y}}{\omega_{z}}}\right)\left(\eta p_{\zeta}+\zeta p_{\eta}\right)+\frac{1}{2}\left(\sqrt{\frac{\omega_{z}}{\omega_{y}}}+\sqrt{\frac{\omega_{y}}{\omega_{z}}}\right)\left(\eta p_{\zeta}-\zeta p_{\eta}\right)$.

The second term can be ignored since it has no matrix elements diagonal in $N$ while the eigenfunctions of the Hamiltonian (19) are rigorously diagonal in $N$. Therefore $j_{x}$ could be replaced by

where

$$
j_{x}=s_{x}+(1+h) l_{\mathrm{tx}}=j_{\mathrm{tx}}+h l_{\mathrm{t} x}
$$

$$
h=\frac{1}{2}\left(\sqrt{\frac{\omega_{z}}{\omega_{y}}}+\sqrt{\frac{\omega_{v}}{\omega_{z}}}\right)-1 .
$$

In the most unfavourable case, $\gamma=30^{\circ}$, the quantity $h$ would have the value

$$
h=(15 / 8 \pi) \beta^{2}+\mathrm{O}\left(\beta^{4}\right) .
$$

Insofar as $h$ can be neglected the matrix elements of $j_{x}$ are independent of the choice of eigenfunctions, and no distinction need be made between the $\left|N l \frac{1}{2} j \Omega\right\rangle$ and the $\left|N l_{\mathrm{t}} \frac{\mathbf{1}}{\mathbf{2}} j_{\mathrm{t}} \Omega_{\mathrm{t}}\right\rangle$ representations.

\section{Electromagnetic Moments and Transition Probabilities}

Expressions for the electromagnetic moments and transition probabilities are straightforward generalizations of the expressions developed for axially symmetric nuclei $2,6,10,11)$. They involve matrix elements of the multipole operators $\mathscr{M}(L, \mu)$ between the possible states $\psi_{K}$ of eq. (3) and summations

t The diagonalization of the Hamiltonian (19) was carried out on the IBM 704 digital computer of The University of Michigan Computing Center. The problem has been programmed for arbitrary values of $C, D, \beta$ and $\gamma$ and computes the eigenvalues and eigenvectors as well as parameters such as $a, b$ etc. and some additional quantities which are useful in the computation of electromagnetic moments and transition probabilities. The diagonalization of the $\left(I+\frac{1}{2}\right) \times\left(I+\frac{1}{2}\right)$ rotational matrices (with $I \leqq \frac{18}{2}$ ) was also carried out on the IBM 704 computer. 
over the coefficients $C_{K}$. The reduced transition probability between states $I_{1}$ and $I_{f}$, governed by an arbitrary tensor operator of rank $L$, is defined as usual by

$$
B\left(L, I_{1} \rightarrow I_{\mathrm{f}}\right)=\sum_{\mu M_{\mathrm{f}}}\left|\left\langle\alpha_{\mathrm{f}} I_{\mathrm{f}} M_{\mathrm{f}}|\mathscr{M}(L, \mu)| \alpha_{1} I_{1} M_{1}\right\rangle\right|^{2}
$$

The $\mu^{\text {th }}$ space-fixed spherical component of the tensor operator is given in terms of the body-fixed components $\mathscr{M}^{\prime}(L, \nu)$ by $\mathscr{M}(L, \mu)=\sum_{\nu} D_{\mu \nu}^{L} \mathscr{M}^{\prime}(L, v)$. If the tensor operator is a function of the odd nucleon's intrinsic coordinates the reduced transition probability can be written

$$
\begin{aligned}
& B\left(L, I_{1} \rightarrow I_{\mathrm{f}}\right)=\left[\sum_{j_{1} j_{\mathrm{f}}} \sum_{K}^{\prime} \sum_{\Omega}^{\prime}\left\langle j_{\mathrm{f}}|| \mathscr{H}^{\prime}(L) \| j_{\mathrm{i}}\right\rangle\left(2 j_{\mathrm{f}}+1\right)^{-\frac{1}{2}}\right. \\
& \times\left\{\sum_{\text {even } \nu} C_{K}^{(\mathrm{i})} C_{(K+\nu)}^{(\mathrm{f})} c_{j_{1} \Omega}^{(\mathrm{i})} c_{j_{\mathrm{f}}(\Omega+\nu)}^{(\mathrm{f})}\left\langle I_{1} L K \nu \mid I_{1} L I_{\mathrm{p}}(K+\nu)\right\rangle\left\langle j_{1} L \Omega v \mid j_{1} L j_{\mathrm{r}}(\Omega+v)\right\rangle\right. \\
& \left.\left.+\sum_{\text {odd } \nu} C_{K}^{(\mathrm{i})} C_{-(K+\nu)}^{(\mathrm{f})} c_{j_{1} \Omega}^{(\mathrm{i})} c_{j_{\mathrm{f}}-(\Omega+\nu)}^{(\mathrm{f})}(-1)^{I_{\mathrm{f}}-j_{\mathrm{f}}}\left\langle I_{1} L K \nu \mid I_{1} L I_{\mathrm{f}}(K+\nu)\right\rangle\left\langle j_{1} L \Omega v \mid j_{1} L j_{\mathrm{f}}(\Omega+\nu)\right\rangle\right\}\right]^{2} .
\end{aligned}
$$

As previously, the prime on the summation symbols indicates that the sums over $K$ and $\Omega$ are restricted to the set of values . . - - $\frac{7}{2},-\frac{3}{2}, \frac{1}{2}, \frac{5}{2}, \frac{9}{2} \ldots$ Only the value of the reduced matrix element of the particle operator of $\operatorname{rank} L$ remains to be calculated. The above formula can be applied specifically if the particle wave functions are expanded in the form of eq. (2), that is in the $\left|N l \frac{1}{2} j \Omega\right\rangle$ representation. For some types of operators the $\left|N l \Lambda \frac{1}{2} \Sigma\right\rangle$ representation used by Nilsson may lead to simpler expressions for the reduced transition probabilities. The quantum number $A$ and $\Sigma$ give the eigenvalues of $l_{z}$ and $s_{z}$, the body-fixed $z$-components of the orbital and spin angular momentum of the odd nucleon, in units of $\hbar$. The Nilsson expansion coefficients $a_{l \Lambda \Sigma}$ are given in terms of the $c_{\boldsymbol{s} \Omega}$ through $\dagger$

$$
a_{l A \Sigma}=\sum_{j}\left\langle l \frac{1}{2} \Lambda \Sigma \mid l \frac{1}{2} j \Omega\right\rangle c_{j \Omega} .
$$

In terms of such coefficients an alternate expression for the reduced transition probability may be useful:

$$
\begin{aligned}
& B\left(L, I_{1} \rightarrow I_{\mathrm{f}}\right) \\
& =\left[\sum _ { \alpha _ { 1 } \alpha _ { \mathrm { f } } } \sum ^ { \prime } \sum _ { \Omega } ^ { \prime } \left\{\sum_{\text {even } \nu} C_{K}^{(\mathrm{i})} C_{(K+\nu)}^{(\mathrm{f})} a_{\alpha_{\mathrm{f}} \Omega}^{(\mathrm{i})} a_{\alpha_{\mathrm{f}}(\Omega+\nu)}^{(\mathrm{f})}\left\langle I_{1} L K \nu \mid I_{1} L I_{\mathrm{f}}(K+\nu)\right\rangle\left\langle a_{\mathrm{f}}(\Omega+v)\left|\mathscr{M}^{\prime}(L, v)\right| \alpha_{i} \Omega\right\rangle\right.\right. \\
& \left.\left.+\sum_{\text {odd } \nu} C_{K}^{(\mathrm{i})} C_{-(K+\nu)}^{(\mathrm{f})} a_{\alpha_{1} \Omega}^{(\mathrm{i})} a_{\alpha_{\mathrm{f}}-(\Omega+\nu)}^{(\mathrm{f})}(-1)^{I_{\mathrm{f}}-\frac{1}{2}}\left\langle I_{1} L K \nu \mid I_{\mathrm{i}} L I_{\mathrm{f}}(K+\nu)\right\rangle\left\langle\alpha_{\mathrm{f}}(\Omega+\nu)\left|\mathscr{M}^{\prime}(L, v)\right| \alpha_{i} \Omega\right\rangle\right\}\right]^{2},
\end{aligned}
$$

where the labels $\alpha$ stand collectively for quantum numbers such as $N, l, A$, and $\Sigma$.

\footnotetext{
+ In order to define the phases of the coefficients it is important to note that the $c_{j \Omega}$ are defined in the $\left|N l \frac{1}{2} j \Omega\right\rangle$ rather than in the $\left|N \frac{1}{2} l j \Omega\right\rangle$ representation. The quantum numbers $N, l$ and $s=\frac{1}{2}$ are never indicated in $c_{j \Omega}$ but are to be implicitly understood.
} 
The expression for the magnetic moment takes the specific form

$$
\begin{aligned}
& \mu=g_{\mathrm{R}} I+\frac{1}{(I+1)} \sum_{K}^{\prime} C_{K}^{2} K\left[\left(g_{s}-g_{\imath}\right)\left\langle s_{0}\right\rangle+\left(g_{\imath}-g_{\mathrm{R}}\right)\langle\Omega\rangle\right] \\
& +\sum_{K}^{\prime} C_{K} C_{-(K-1)}(-1)^{I-\frac{1}{2}} \frac{[(I+K)(I-K+1)]^{\frac{1}{2}}}{2(I+1)}\left[\left(g_{s}-g_{l}\right)\left\langle s_{-}\right\rangle+\left(g_{l}-g_{\mathrm{R}}\right)(a+b)\right] \\
& +\sum_{K}^{\prime} C_{K} C_{-(K+1)}(-1)^{I-\frac{1}{2}} \frac{[(I-K)(I+K+1)]^{\frac{1}{t}}}{2(I+1)}\left[\left(g_{s}-g_{\imath}\right)\left\langle s_{+}\right\rangle+\left(g_{\imath}-g_{\mathrm{R}}\right) c\right],
\end{aligned}
$$

where $(a+b)$ and $c$ are written out explicitly in eqs. (10) and (11), while $\langle\Omega\rangle=$ $\Sigma_{j \Omega}^{\prime} c_{j \Omega}^{2} \Omega$. Expectation values such as $\left\langle s_{0}\right\rangle$ have their simplest form in terms of the expansion coefficients $a_{l \Lambda \Sigma}$ :

$$
\begin{aligned}
& \left\langle s_{0}\right\rangle=\frac{1}{2} \sum_{l} \sum_{\Omega}^{\prime}\left(a_{l\left(\Omega-\frac{1}{2}\right) \frac{1}{2}}^{2}-a_{l(\Omega+1)-\frac{1}{l}}^{2}\right), \\
& \left\langle s_{-}\right\rangle=\sum_{l} \sum_{\Omega}^{\prime}(-1)^{l} a_{l\left(\Omega-\frac{1}{2}\right) !} a_{l,-\left(\Omega-\frac{1}{2}\right) \frac{1}{2}}, \\
& \left\langle s_{+}\right)=\sum_{l} \sum_{\Omega}^{\prime}(-1)^{l} a_{l(\Omega+1),-\frac{1}{l}} a_{l,-\left(\Omega+\frac{1}{2}\right),-\frac{1}{l}} .
\end{aligned}
$$

The electric quadrupole moment is given by

$$
\begin{aligned}
& Q_{\mathrm{s}}=\left(Q_{0} \cos \gamma+q_{0}\right) \sum_{K}^{\prime} C_{K}^{2} \frac{\left[3 K^{2}-I(I+1)\right]}{(I+1)(2 I+3)} \\
& +\left(Q_{0} \sin \gamma+q_{2}\right) \sum_{K}^{\prime} C_{K} C_{(K-2)} \frac{[3(I+K)(I+K-1)(I-K+1)(I-K+2)]^{1}}{(I+1)(2 I+3)},
\end{aligned}
$$

where $Q_{0}$ gives the contribution of the deformed core

$$
Q_{0}=3 Z e R_{0}{ }^{2} \beta / \sqrt{ } 5 \pi,
$$

while $q_{0}$ and $q_{2}$ give the contribution of the odd nucleon. The matrix elements of $e_{p} v_{p}^{2} Y_{2 v}\left(\theta_{p}\right)$ for the odd particle are again evaluated most easily in terms of the expansion coefficients $a_{l \Lambda \Sigma}$ and lead to

$$
\begin{aligned}
q_{0}= & \frac{2 e_{\mathbf{p}} \hbar}{m \omega_{0}} \sum_{l \Lambda}\left[-a_{l \Lambda}^{2}\left(N+\frac{3}{2}\right)\left[\frac{l(l+1)}{(2 l-1)(2 l+3)}\right]^{\frac{1}{2}}\langle l 2 \Lambda 0 \mid l 2 l \Lambda\rangle\right. \\
& \left.+2 a_{l \Lambda} a_{(l-2) \Lambda}\left[\frac{(N+l+1)(N-l+2) 3 l(l-1)}{2(2 l-1)(2 l-3)}\right]^{\frac{1}{2}}\langle l 2 \Lambda 0 \mid l 2(l-2) \Lambda\rangle\right] \\
q_{2}= & \frac{2 e_{\mathbf{p}} \hbar}{m \omega_{0}} \sum_{l \Lambda}\left[-a_{l \Lambda} a_{l l \Lambda+2)}\left(N+\frac{3}{2}\right)\left[\frac{l(l+1)}{(2 l-1)(2 l+3)}\right]^{\frac{1}{2}}\langle l 2 \Lambda 2 \mid l 2 l(\Lambda+2)\rangle\right. \\
& +a_{l \Lambda} a_{(l-2)(\Lambda+2)}\left[\frac{(N+l+1)(N-l+2) 3 l(l-1)}{(2 l-1)(2 l-3)}\right]^{\frac{1}{2}}\langle l 2 \Lambda 2 \mid l 2(l-2)(\Lambda+2)\rangle \\
& \left.+a_{l \Lambda} a_{(l+2)(\Lambda+2)}\left[\frac{(N+l+3)(N-l) 3(l+2)(l+1)}{(2 l+3)(2 l+5)}\right]^{\frac{1}{2}}\langle l 2 \Lambda 2 \mid l 2(l+2)(\Lambda+2)\rangle\right] .
\end{aligned}
$$


The quantum number $\Sigma$ (as well as the harmonic oscillator quantum number $N$ ) is not indicated explicitly in the coefficients $a_{l \Lambda}$ since all the matrix elements are diagonal in $\Sigma$. (The quantity $e_{\mathbf{p}}$ is the charge of the odd nucleon. An effective charge might be used if vibrational interactions with the core are taken into account.) It should be noted that $Q_{\mathrm{s}}$ can be negative even if the nucleus has a large positive intrinsic deformation $\left(\beta>0, \gamma \leqq 30^{\circ}\right)$.

Since explicit expressions for $\mathrm{Ml}$ and $\mathrm{E} 2$ reduced transition probabilities may be useful, they are given below for transitions $I_{1}$ to $I_{\mathbf{l}}$ between different rotational states based on the same particle excitation:

$$
\begin{aligned}
& B\left(\mathrm{Ml}, I_{1} \rightarrow I_{\mathrm{f}}\right)=\frac{3}{4 \pi}\left[g_{\mathrm{R}}\left[I_{1}\left(I_{\mathrm{1}}+1\right)\right] \delta_{I_{1} I_{\mathrm{f}}} \sum_{K}^{\prime} C_{K}{ }^{\left({ }^{1)}\right.} C_{K}{ }^{(\mathrm{t})}\right. \\
& +\sum_{K}^{\prime} C_{K}^{(1)} C_{K}^{(\mathfrak{l})}\left\langle I_{1} 1 K 0 \mid I_{1} 1 I_{\mathrm{f}} K\right\rangle\left\{\left(g_{s}-g_{l}\right)\left\langle s_{0}\right\rangle+\left(g_{l}-g_{\mathrm{R}}\right)\langle\Omega\rangle\right\} \\
& +\sum_{K}^{\prime} C_{K}^{(1)} C_{-(K-1)}^{(f)}\left\langle I_{1} 1 K-1 \mid I_{1} 1 I_{1}(K-1)\right\rangle \frac{(-1)^{I_{1}-\frac{1}{2}}}{\sqrt{ } 2}\left\{\left(g_{s}-g_{l}\right)\left\langle s_{-}\right\rangle+\left(g_{l}-g_{R}\right)(a+b)\right\} \\
& \left.+\sum_{K}^{\prime} C_{K}^{(1)} C_{-(K+1)}^{(f)}\left\langle I_{1} 1 K 1 \mid I_{1} 1 I_{\mathrm{f}}(K+1)\right\rangle \frac{(-1)^{I_{\mathrm{f}}+\mathrm{t}}}{\sqrt{ } 2}\left\{\left(g_{s}-g_{l}\right)\left\langle s_{+}\right\rangle+\left(g_{l}-g_{\mathrm{R}}\right) c\right\}\right]^{2} .
\end{aligned}
$$

(If there is a change in the particle excitation of the odd nucleon in the transition, the expressions for the Ml transition probabilities are merely slight generalizations of the above formula which are obtained if products of the form $c_{j \Omega} c_{j,-(\Omega-1)}$ and $a_{l(\Omega-1) i} a_{l,-(\Omega-1)\}}$ in the expectation values are replaced by $c_{j \Omega}^{(\mathrm{i})} c_{j,-(\Omega-1)}^{(\mathrm{f})}$ and $a_{l\left(\Omega-\frac{1}{2}\right)}^{(\mathrm{i})} a_{l-(\Omega-\mathbf{i})\}}^{(\mathrm{f})}$, respectively.)

The $E 2$ rates, again for transitions between different rotational states based on the same particle excitation, are given by

$$
\begin{aligned}
B\left(\mathrm{E} 2, I_{1} \rightarrow I_{\mathrm{l}}\right)= & \frac{5}{16 \pi}\left[\left(Q_{0} \cos \gamma+q_{0}\right) \sum_{K}^{\prime} C_{K}^{(1)} C_{K}^{(\mathrm{f})}\left\langle I_{1} 2 K 0 \mid I_{1} 2 I_{\mathrm{f}} K\right\rangle\right. \\
& +\frac{\left(Q_{0} \sin \gamma+q_{2}\right)}{\sqrt{ } 2} \sum_{K}^{\prime}\left(C_{K}^{(1)} C_{(K-2)}^{(\mathrm{f})}\left\langle I_{1} 2 K-2 \mid I_{1} 2 I_{\mathrm{f}}(K-2)\right\rangle\right. \\
& \left.\left.+C_{K}^{(1)} C_{(K+2)}^{(\mathrm{f})}\left\langle I_{1} 2 K 2 \mid I_{1} 2 I_{\mathrm{f}}(K+2)\right\rangle\right)\right]^{2}
\end{aligned}
$$

(If the transition involves a change in the particle excitation, the core can give no contribution. The contributions of the odd nucleon will have a more complicated form and will involve five different terms of the form $q_{2}, q_{-2}$, $q_{1}, q_{-1}$ and $\left.q_{0}\right)$.

\section{Spectroscopic Factors for Nucleon Emission or Capture}

In this section we consider the relative amplitudes for nucleon capture into, or removal from, bound states of asymmetric nuclei. Such amplitudes are 
measured by stripping and pick-up reactions, respectively ${ }^{\mathbf{1 2}}$ ). The amplitude for stripping or pick-up contains an overlap integral over target and residual nucleus wave functions, which may be expanded in the form

$$
\int \psi_{A+1}^{*}(\xi, \mathbf{x}) \psi_{A}(\xi) \mathrm{d} \xi=\sum_{j} \beta_{j}\left\langle I_{A} j M_{A} m \mid I_{A} j I_{A+1} M_{A+1}\right\rangle \chi_{m}^{j}(\mathbf{x}),
$$

where $\mathbf{x}$ denotes the coordinates of the $(A+1)$ th nucleon, while the $z$-component $m$ refers to a space-fixed coordinate system. Quantum numbers such as $N$ and $l$ are not explicitly indicated in eq. (30) for simplicity. The parentage coefficient $\beta_{j}$ in expansion $(30)$ is then the amplitude for finding the $(A+1)$ th nucleon with angular momentum $j$ within the nucleus of mass $A+1$ when in a state $\psi_{A+1}$, with the remaining nucleus of mass $A$ in state $\psi_{A}$. If there are $n$ nucleons of this type in nucleus $(A+1)$, the capture or emission probability is proportional to the spectroscopic factor ${ }^{12}$ )

$$
S(j)=n\left|\beta_{j}\right|^{2}
$$

The two cases we shall consider are the removal of a nucleon (i) from an odd-mass nucleus to leave an even nucleus, and (ii) from an even nucleus to leave an odd-mass nucleus. (The stripping processes are simply the inverses.) In previous sections we have only considered the states of a nucleus with a single nucleon coupled to a structureless asymmetric core. This is adequate for the description of the removal of the nucleon if the core is identified with the resulting even nucleus. However, the removal of a nucleon from an even nucleus requires a more detailed description. One simple generalization is to consider the nucleons to fill (consistently with the Pauli principle) the lowest levels in the ellipsoidal single-particle potential well. If we restrict ourselves to configurations in which even numbers of nucleons are paired off in the lowest (doubly degenerate) single-particle levels while only the last odd particle is allowed to change its state, it is straightforward to show that the resulting energy levels and wave functions are the same as for the single-particle model of the preceding sections. (This simple equivalence does not hold for more complicated configurations.) The other nucleons merely form an inert core which we assume to be the same as the corresponding even nucleus. However, we now have a consistent mechanism for removal of a nucleon from an even nucleus, or inversely its addition to an odd-mass nucleus.

With this assumption, the wave-function of the even nucleus now has the form

$$
\psi_{\mathrm{e}}=\sqrt{\frac{2 I_{\mathrm{e}}+1}{16 \pi^{2}}} \sum_{K_{\mathrm{e}}}^{\prime} C_{K_{\mathrm{e}}}\left[D_{M_{\mathrm{e}} K_{\mathrm{e}}}^{I_{\mathrm{e}}}+(-1)^{I_{\mathrm{e}}} D_{M_{\mathrm{e}}-K_{\mathrm{e}}}^{I_{\mathrm{e}}}\right] X .
$$

The summation is over positive, even values of $K_{\mathrm{e}}$ only, including zero, and $C_{0}$ is chosen to absorb a factor $\sqrt{ } 2$ which arises because the normalization of the 
function in brackets is different for the special case $K_{\mathrm{e}}=0$. The $X$ is a normalized determinant of the wave functions (2) for the occupied single-particle states ${ }^{\dagger}$. The odd-mass nucleus has a wave function which is a generalization of (3),

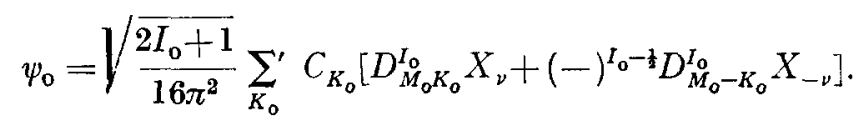

The $X_{\nu}$ is again a determinant of single-particle states. For the configurations which we are considering, all but one of the nucleons are paired off, while the odd one occupies the state $\chi_{\nu}$. The $X_{-\nu}$ is similar but with the odd nucleon in the state $\chi_{-\nu}$.

With these wave-functions the amplitudes $\beta$ from the overlap (30) are the following ${ }^{+\dagger}$ :

Case (i), $A=$ even:

$$
\begin{aligned}
\sqrt{n} \beta_{j}=\sqrt{\frac{\sqrt{2 I_{\mathrm{e}}+1}}{2 I_{\mathrm{o}}+1} \sum_{K_{\mathrm{e}}}^{\prime} \sum_{K_{\mathrm{o}}}^{\prime} C_{K_{\mathrm{o}}}^{(A)} C_{K_{\mathrm{e}}}^{(A+1)}\left[c_{j\left(K_{\mathrm{o}}-K_{\mathrm{e}}\right)}\left\langle I_{\mathrm{e}} j K_{\mathrm{e}}\left(K_{\mathrm{o}}-K_{\mathrm{e}}\right) \mid I_{\mathrm{e}} j I_{\mathrm{o}} K_{\mathrm{o}}\right\rangle\right.} \\
\left.+(-1)^{I_{\mathrm{e}}} c_{j\left(K_{\mathrm{o}}+K_{\mathrm{e}}\right)}\left\langle I_{\mathrm{e}} j-K_{\mathrm{e}}\left(K_{\mathrm{o}}+K_{\mathrm{e}}\right) \mid I_{\mathrm{e}} j I_{\mathrm{o}} K_{\mathrm{o}}\right\rangle\right] .
\end{aligned}
$$

Case (ii), $A=$ odd:

$$
\begin{aligned}
& \sqrt{n \beta_{j}}=\sqrt{\frac{2 I_{\mathrm{o}}+1}{2 I_{\mathrm{e}}+1}} \sum_{K_{\mathrm{o}}}^{\prime} \sum_{K_{\mathrm{e}}}^{\prime} C_{K_{\mathrm{o}}}^{(A)} C_{K_{\mathrm{e}}}^{(A+1)}(-1)^{j-1}\left[c_{j\left(K_{\mathrm{o}}-K_{\mathrm{e}}\right)}\left\langle I_{\mathrm{o}} j K_{\mathrm{o}}\left(K_{\mathrm{e}}-K_{\mathrm{o}}\right) \mid I_{\mathrm{o}} j I_{\mathrm{e}} K_{\mathrm{e}}\right\rangle\right. \\
& \left.+(-1)^{I_{\mathrm{e}}} c_{j\left(K_{\mathrm{o}}+K_{\mathrm{e}}\right.}\left\langle I_{\mathrm{o}} j K_{\mathrm{o}}-\left(K_{\mathrm{e}}+K_{\mathrm{o}}\right) \mid I_{\mathrm{o}} j I_{\mathrm{e}}-K_{\mathrm{e}}\right\rangle\right] \text {, }
\end{aligned}
$$

where $n$ in (34) or (35) is the number of nucleons, in the total system of $(A+1)$ nucleons, which are of the same type as the one removed.

The spectroscopic factor is then $S(j)=\left|\sqrt{n} \beta_{j}\right|^{2}$.

\section{Numerical Results and Possible Applications}

According to our model the odd-mass nucleus is assumed to have a well defined asymmetric equilibrium shape. In looking for possible examples for such a model the neighbouring even nuclei might be used as a guide. Preferably these should have large asymmetry and show relatively little vibrationrotation interaction. There is some indication that nuclei around $A=190$ may satisfy both requirements. Asymmetries of $16.5^{\circ}, 19^{\circ}, 22^{\circ}$ and $25^{\circ}$ have been reported ${ }^{3}$ ) for the sequence $\mathrm{Os}^{186}, \mathrm{Os}^{188}, \mathrm{Os}^{190}$ and $\mathrm{Os}^{192}$, for example; and the indications are that asymmetric rotator theory is satisfactory, particu-

\footnotetext{
† If neutrons and protons are treated as distinguishable, $X$ is a product of determinants of order $N$ for the neutrons and of order $Z$ for the protons.

${ }^{+t}$ The indices e and o refer to the even and odd-mass nucleus, respectively.
} 
larly for the isotopes $\mathrm{Os}^{190}$ and $\mathrm{Os}^{192}$. For this reason it was decided to make a preliminary survey of the odd-mass nuclei around $A=190$. Unfortunately very little is known about the excited states of the odd-mass isotopes of osmium, although it might be noted that the experimentally observed magnetic moment of $\mathrm{Os}^{187}(\mu=0.12)$ is considerably smaller than the value predicted on the

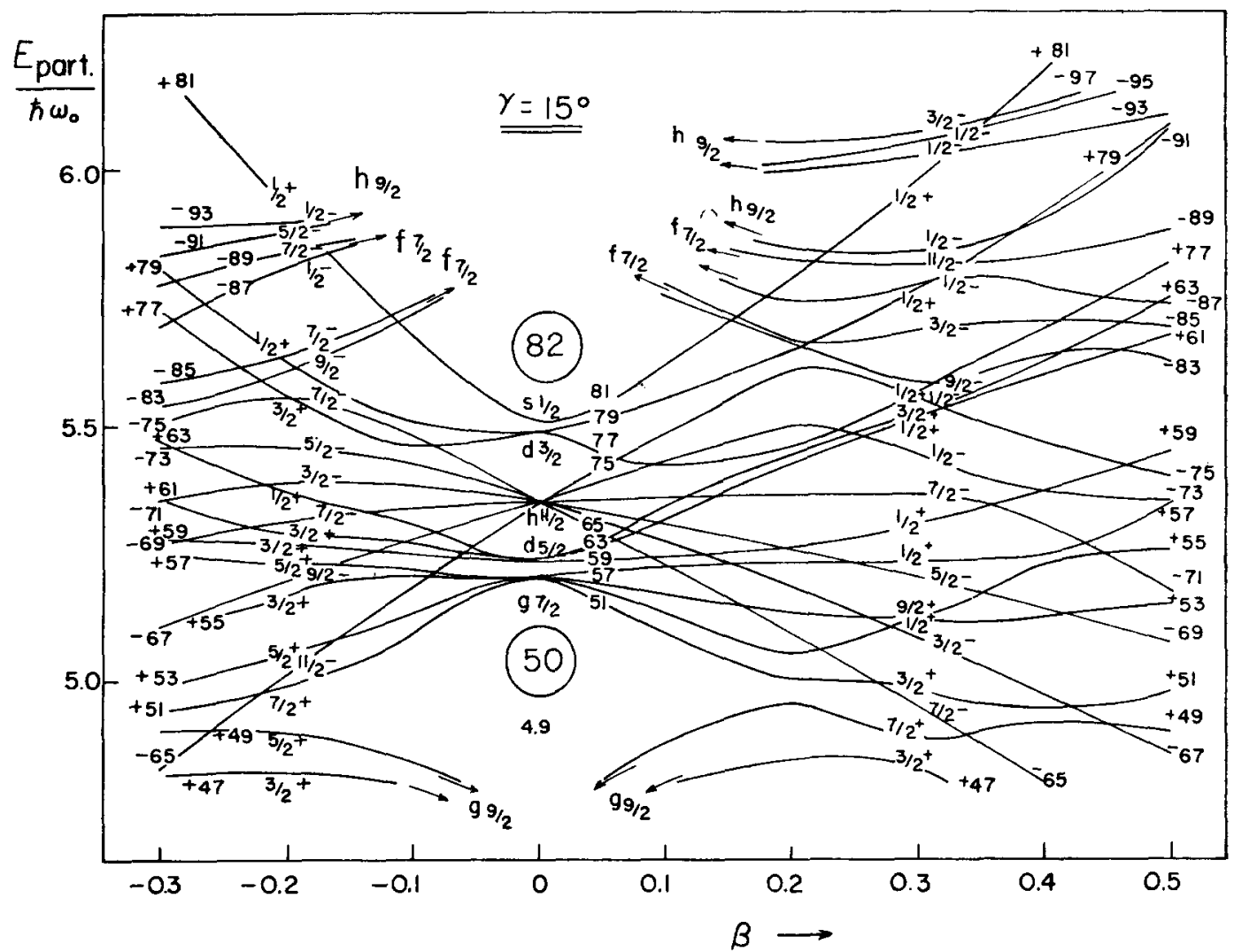

Fig. 1. Single-particle levels for odd-proton nuclei in the region $50<Z<82\left(\gamma=15^{\circ}\right)$. The parameters $C(\kappa)$ and $D(\mu)$ for these levels have been chosen to have the values recommended by Mottelson and Nilsson ${ }^{\circ}$ ). The calculated wave functions correspond to the parameters $C=$ $-0.1(\kappa=0.05)$; and $D=-0.0275(\mu=0.55)$ for $N=4(+$ parity $)$ levels, while $D=-0.0250$ $(\mu=0.50)$ for $N=5$ ( - parity) levels. Since levels of a given parity do not cross, the single particle levels are labelled by their parity and by an ordering number which would be the number of the odd proton if the single-particle levels, at small deformation $\beta$, were filled in order. The single-particle levels are also labelled with the spin and parity of the lowest rotational level (ground state) for the specific deformations $\beta=0.3$ and $\beta=-0.2$.

basis of an axially symmetric rotator model $\left(\mu=0.8\right.$, ref. $\left.{ }^{9}\right)$, table 7$)$. Although insufficient experimental information is available for a test of asymmetric rotator theory in the case of $\mathrm{Os}^{187}$ and $\mathrm{Os}^{189}$, the experimental situation is somewhat more favourable in the case of some of the isotopes of Re, Ir, Au, Pt and perhaps $W$. 


\subsection{ODD-PROTON NUCLEI}

The low-lying levels of the isotopes of $\operatorname{Re}$ seem to form a $\frac{5}{2}+, \frac{7}{2}+, \frac{9}{2}+$ rotational sequence. The various isotopes of Ir and Au all have $\frac{3}{2}+$ ground states with magnetic moments between 0.1 and 0.2 nuclear magnetons. On the basis of an axially symmetric model rotational bands based on $\Omega=\frac{5}{2}+$

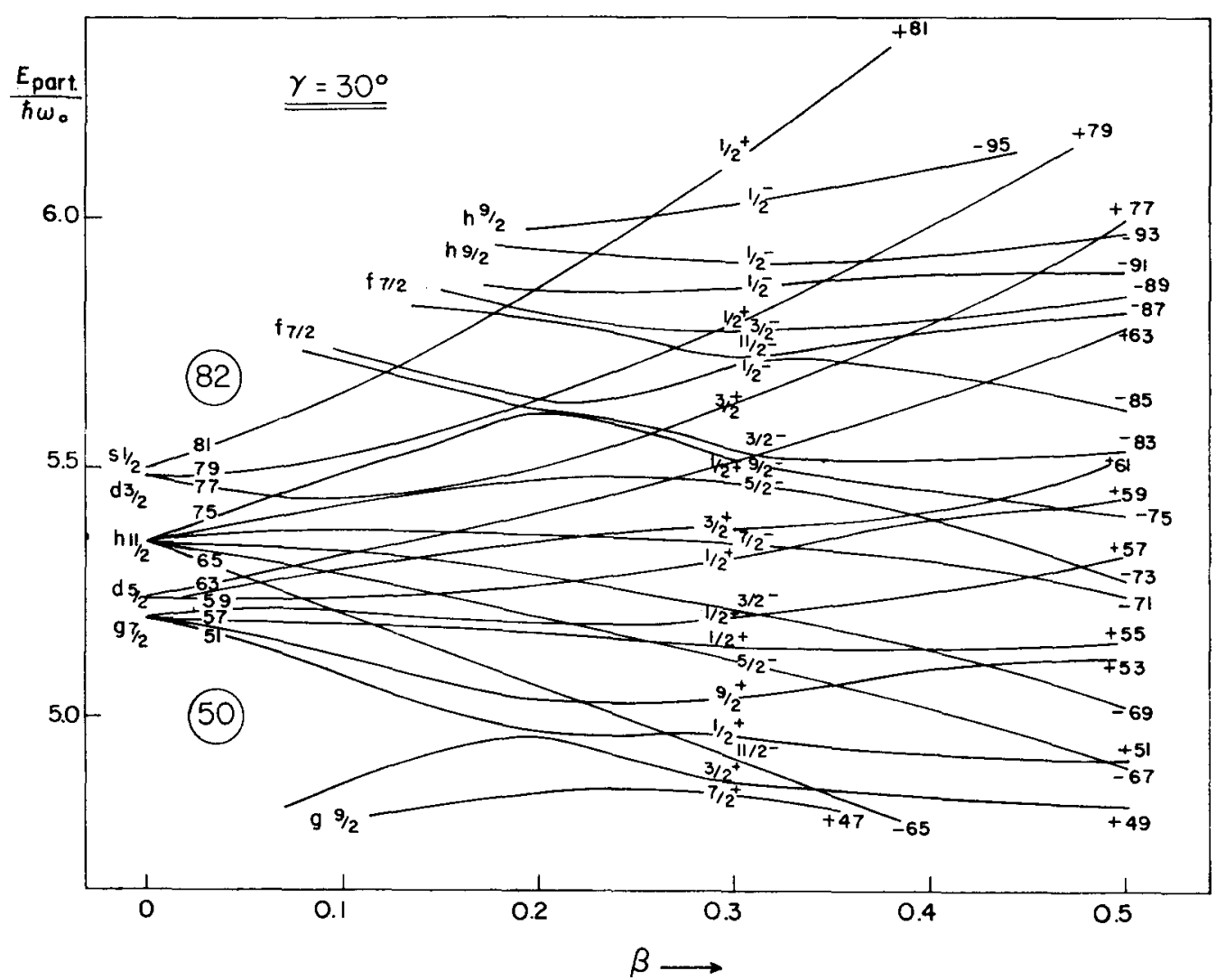

Fig. 2. Single-particle levels for odd-proton nuclei in the region $50<Z<82\left(\gamma=30^{\circ}\right)$. See caption for fig. 1 .

and $\frac{\mathbf{3}}{\mathbf{2}}+$ states should be expected in these two cases. The most likely particle states are the [402] $\Omega=\frac{\mathbf{5}}{\mathbf{2}}+$ Nilsson level for the $75^{\text {th }}$ proton in Re and the [402] $\Omega=\frac{3}{2}+$ Nilsson level for the $\mathbf{7 7}^{\text {th }}$ and $\mathbf{7 9}^{\text {th }}$ protons in Ir and Au. (See fig. 3 , ref. $\left.{ }^{9}\right)$ ). The levels are labelled by the asymptotic quantum numbers $\left[N n_{z} \Lambda\right.$ ].) In an asymmetric nucleus $\Omega$ is no longer a good quantum number. With the introduction of a small asymmetry Nilsson levels with different $\Omega$ interact with each other. As a result the single particle levels of an asymmetric nucleus cannot cross each other when plotted as a function of the deformation parameter $\beta$, except for states of opposite parity. For small values of the 
asymmetry parameter $\gamma$, the single particle energies differ little from the Nilsson values, except in regions where Nilsson levels with different $\Omega$ cross each other or lie close together over large ranges of $\beta$. The single particle levels for asymmetric odd-proton nuclei in the region $50<Z<82$ are shown in fig. 1 for an intermediate value of the asymmetry parameter $\gamma=15^{\circ}$ and in fig. 2 for the largest possible asymmetry $\gamma=30^{\circ}$. In order to give some idea of the possible rotational bands which can be built on these particle states, the levels have been labelled with the spin of the ground state (lowest rotational

TABLE 2

Order of low-lying rotational levels for odd-proton nuclei ${ }^{\text {) }}$

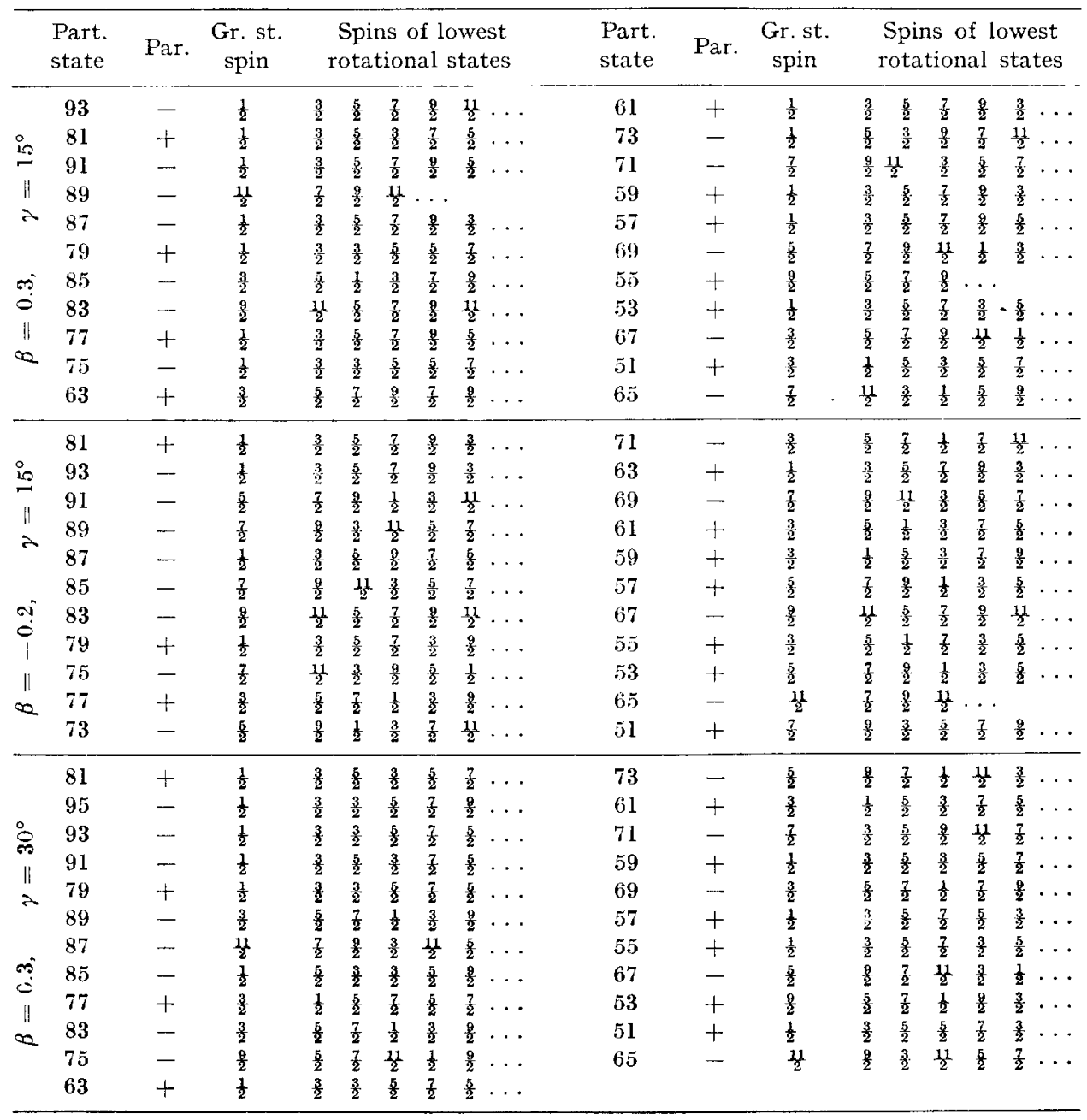

a) Levels with $I \leqq \frac{11}{2}$ are listed for the negative parity states, $I \leqq \frac{8}{2}$ for the positive parity staies. 
level), for $\beta$ values of -0.2 and 0.3 . For these specific values of $\beta$ and $\gamma$ the spin values of the lowest rotational levels based on each particle state are also listed in table 2 starting with the ground state at the left. Since the rotational spin sequences may be very sensitive functions of both $\beta$ and $\gamma$, the spin values listed in table 2 can only serve as an indication of the types of rotational sequences to be expected. The investigation of any specific example must be based on a plot of the rotational energies as a function of $\gamma$ for appropriate values of $\beta$. To get some understanding of the behaviour of the rotational levels, however, consider particle state 65 , the lowest negative parity singleparticle state which grows out of the $h_{\frac{11}{2}}$ shell model level, as an example. In the prolate symmetric rotator limit, $\beta>0, \gamma=0^{\circ}$, this is a pure $\Omega=\frac{1}{2}$ state with an $I=\frac{\mathbf{3}}{\mathbf{2}}$ rotational ground state. In the oblate symmetric rotator limit, $\beta<0, \gamma=0^{\circ}$, or what is equivalent, $\beta>0, \gamma=60^{\circ}$, this is a pure $\Omega=\frac{11}{2}$ state with an $I=\frac{11}{2}$ rotational ground state. Between $\gamma$ of $0^{\circ}$ and $60^{\circ}$ $(\beta>0)$ the ground states therefore changes from an $I=\frac{3}{2}$ to an $I=\frac{11}{2}$ state. Note, however, that for $\gamma=30^{\circ}$ an $I=\frac{11}{2}$ value has already been reached, whereas for $\gamma=15^{\circ}$ the ground state $I$ value is $\frac{7}{2}$. Note also from fig. 2 that $I=\frac{1}{2}$ ground states are very common in the case of large asymmetry. Although the single particle levels could be labelled with the asymptotic quantum numbers $\left[n_{x} n_{y} n_{z}\right]$ this does not seem to be a useful label since the order of the single particle levels $\left[n_{x} n_{y} n_{z}\right]$ would be a function of $\gamma$. Since levels of the same parity cannot cross each other, the levels are labelled by the occupation of the odd nucleon. Since levels of opposite parity do cross each other, however, such a scheme cannot give the order of the single particle levels for all values of $\beta$. In figs. 1 and 2 the particle levels are therefore labelled by the number of the odd nucleon $(Z)$, for the case of very small deformation $\beta$. Thus, level 81 grows out of the $s_{\frac{1}{2}}$ shell model state, while levels 79 and 77 grow out of the $\mathrm{d}_{\frac{3}{2}}$ shell model state. For small values of $\beta$ the wave function for the state $\mathbf{7 7}$ becomes predominantly $\Omega=\frac{1}{2}$ as $\gamma$ approaches zero. For larger values of $\beta$, however, the wave function for state 77 approaches that of a pure $\Omega=\frac{\mathbf{5}}{\mathbf{2}}$ state as $\gamma$ approaches zero. (Note thaı the Nilsson levels [411] $\Omega=\frac{1}{2}+$ and [402] $\Omega=\frac{5}{2}+$ cross each other at $\beta \approx 0.19$ ). The state 77 may therefore be expected to be the state of the odd proton in the isotopes of Re if asymmetric rotator theory is applicable.

Fig. 3 shows the rotational energies based on the particle state 77 as a function of $\gamma$ for relatively small asymmetry and a value of $\beta(=0.3)$ which reproduces the experimentally observed value of the electric quadrupole moment for $\mathrm{Re}^{\mathbf{1 8 5}}$. The behaviour of these rotational energy levels as a function of $\gamma$ is characteristic of a large number of particle states. For very small values of $\gamma$ the particle wave function is predominantly $\Omega=\frac{5}{2}$. The lowest set of rotational states form an $I=\frac{5}{2}, \frac{7}{2}, \frac{9}{2}, \ldots$ sequence with approximate $I(I+1)$ spacing. The corresponding wave functions are almost pure $K=\frac{5}{2}$ rotational functions 
(corresponding to $K_{\mathrm{R}}=0$ ). At $\gamma=10^{\circ}$, for example, the low $I=\frac{\mathbf{5}}{\mathbf{2}}$ state is $99.98 \%$ pure $K=\frac{5}{2}$. The particle wave function, on the other hand, is only $80.7 \%$ pure $\Omega=\frac{5}{2}$, with the following admixtures: $\Omega=\frac{1}{2}: 10.1 \%, \Omega=$ $-\frac{7}{2}: 7.3 \%, \Omega=\frac{9}{2}: 1.9 \%, \Omega=-\frac{3}{2}: 0.1 \%$. The rotational energies of these states first increase with $\gamma$ since the energies are very sensitive to a small amount of admixture of $K_{\mathrm{R}}=2$ near $\gamma=0^{\circ}$ through the $A_{3}\left\langle K_{\mathrm{R}}{ }^{2}\right\rangle$ term in the rota-

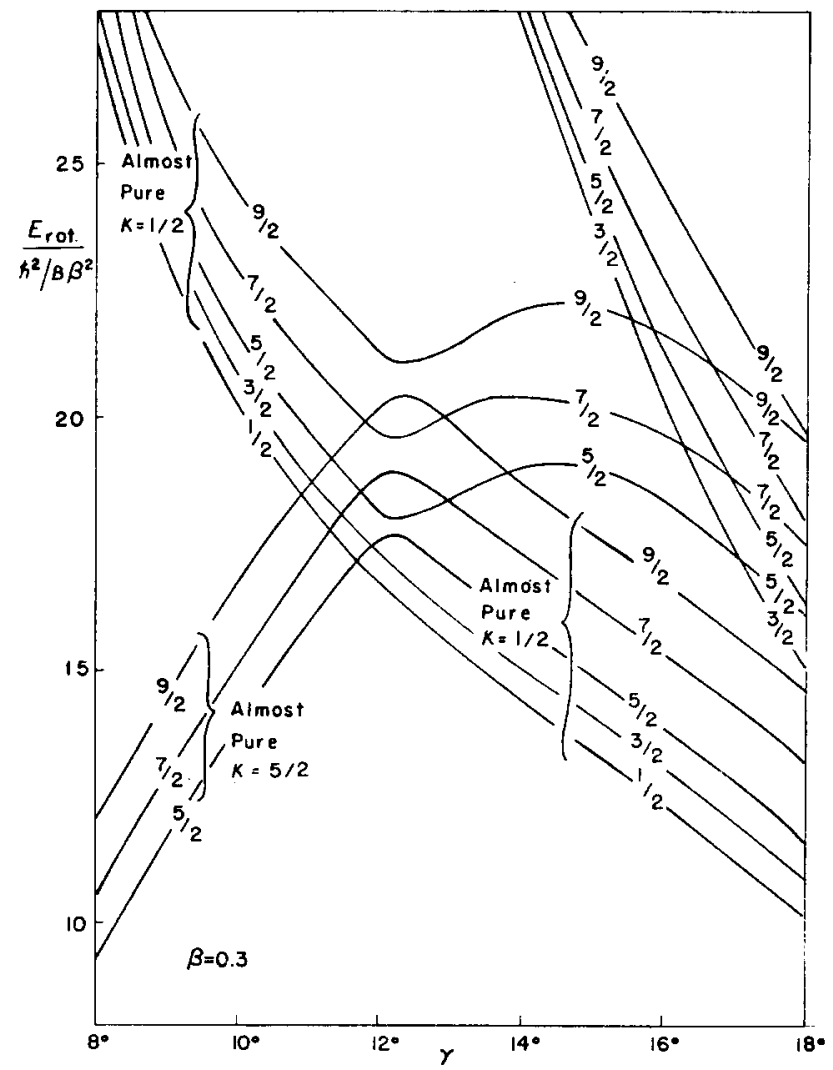

Fig. 3. Rotational energies for the particle state $77(\beta=0.3)$. As $\gamma$ approaches $0^{\circ}$, particle state 77 goes over to the axially symmetric [402] $\Omega=\frac{5}{2}+$ particle state for this deformation

tional Hamiltonian; $A_{3}$ is inversely proportional to $\sin ^{2} \gamma$. In the axially symmetric limit the $I=\frac{5}{2}$ ground state has a rotational energy of 0.96 in the units of $\hbar^{2} / B \beta^{2}$ appropriate to fig. 3. A relatively large zero point rotational energy, such as that predicted for $\gamma \approx 12^{\circ}$, may be important in determining the order of rotational bands based on different particle excitations. For $\gamma<11^{\circ}$ the next set of rotational levels form and $I=\frac{1}{2}, \frac{3}{2}, \frac{5}{2}, \ldots$ sequence with $K_{\mathrm{R}}$ of approximately 2 units. As $\gamma$ approaches $0^{\circ}$ these rotational levels rapidly go to large values through the influence of the $A_{3}\left\langle K_{\mathrm{R}}{ }^{2}\right\rangle$ term. At $\gamma=12^{\circ}$ the $I=\frac{1}{2}$ and $\frac{3}{2}$ levels form the lowest rotational states. At $\gamma \approx 12.5^{\circ}$ the states with 
$I \geqq \frac{\mathbf{5}}{\mathbf{2}}$ have rotational wave functions with almost equal proportions of $K=\frac{\mathbf{5}}{\mathbf{2}}$ and $K=\frac{1}{2}$. By $\gamma \approx 14^{\circ}$ to $15^{\circ}$ the $I=\frac{1}{2}, \frac{3}{2}, \frac{5}{2}, \ldots$ sequence has crossed over the $I=\frac{5}{2}, \frac{7}{2}, \frac{9}{2}, \ldots$ sequence. (Note, however, that levels of the same $I$ do not actually cross.) The lower sequence now has rotational wave functions of almost pure $K=\frac{1}{2}$ character while the higher sequence is predominantly $K=\frac{\mathbf{5}}{2}$. At $14^{\circ}$ the particle wave function consists of the following admixtures: $\Omega=\frac{5}{2}: 62.6 \%, \Omega=-\frac{7}{2}: 21.5 \%, \Omega=\frac{9}{2}: 3.0 \%, \Omega=\frac{1}{2}: 12.8 \%, \Omega=-$ $\frac{3}{2}: 0.1 \%$. At still larger values of $\gamma$ an $I=\frac{3}{2}, \frac{5}{2}, \frac{7}{2}, \ldots$ sequence with $K_{\mathrm{R}}$ approximately equal to 4 units crosses the $I=\frac{5}{2}, \frac{7}{2}, \frac{9}{2}, \ldots$ sequence and near $\gamma$ of $18^{\circ}$ the higher levels have strongly mixed $K=\frac{5}{2}$ and $K=-\frac{3}{2}$ rotational wave functions.

The observed levels of $\mathrm{Re}^{185}$ consist of the following: an $I=\frac{5}{2}^{+}, \frac{7}{2}^{+}, \frac{9}{2}^{+}$ sequence built on the ground state at 0,128 , and $287 \mathrm{keV}$; a $\frac{1}{2}+, \frac{3}{2}+$ doublet at 646 and $717 \mathrm{keV}$; and another $\frac{1}{2}+\frac{3}{2}+$ doublet at 872 and $879 \mathrm{keV}$ (with the spin assignment of these last two somewhat uncertain). Mottelson and Nilsson on the basis of the axially symmetric model ${ }^{9}$ ) explain these as the beginning of rotational bands based on the single particle Nilsson levels [402] $\Omega=\frac{5}{2}+$, [400] $\Omega=\frac{1}{2}+$, and [411] $\Omega=\frac{1}{2}+$, in that order. In terms of the asymmetric model, however, the possibility exists that two of these three rotational sequences are based on the same single particle level (77). With $\gamma \approx 10^{\circ}$ the spacing of the first five rotational levels corresponds to the observed energies. Since the two apparent rotational sequences at $10^{\circ}$ correspond to almost pure $K=\frac{\mathbf{5}}{\mathbf{2}}$ and $K=\frac{1}{\mathbf{2}}$ bands even though $K$ is not a good quantum number in the asymmetric case, it would be difficult to distinguish between an axially symmetric and an asymmetric model. The E2 rates would be very similar in the two cases. Predictions for the magnetic dipole moment differ somewhat since the asymmetric ground state particle wave function is not a pure $\Omega=\frac{\mathbf{5}}{\mathbf{2}}$ wave function; but these differences are small. The theoretical value on the basis of the asymmetric model is $\mu=\mathbf{3 . 3 7}$ (for $\beta=0.3, \gamma=10^{\circ}$ ), compared with an experimental value of 3.16 , and a theoretical value of 3.7 predicted on the basis of the axially symmetric model ${ }^{9}$ ). The major differences between the theoretical predictions for the two models would probably involve the transition probabilities between the $\frac{3}{2}+, \frac{1}{2}+$ levels and the ground state band. According to the axially symmetric model these transitions would involve a change in the odd nucleon particle excitation. In the asymmetric model, however, transitions from one $\frac{1}{2}+, \frac{3}{2}+$ doublet to the ground state would involve no change in the particle wave function. At present there is not sufficient experimental information to decide between the two possibilities. Note that the selection rule $|\Delta K| \leqq 1$ would inhibit the Ml rates between two rotational sequences in the case of both models. Note also that the collective contributions to the $\mathrm{E} 2$ rates are small for a $\Delta K \approx 2$ transition in the asymmetric model if $\sin \gamma$ is very small. 
The rotational energies in fig. 3 have been drawn only for $\gamma \leqq 18^{\circ}$. Near $\gamma \approx 20^{\circ}$ the single particle levels 77 and 63 have very nearly the same energy so that diagonalization of $\left(I+\frac{1}{2}\right) \times\left(I+\frac{1}{2}\right)$ rotational matrices based on a single particle state cannot be expected to give sensible values for the rotational energies. For $\gamma>22.5^{\circ}$ the difference in energy between the particle states $\mathbf{7 7}$ and $\mathbf{6 3}$ is again large enough so that the rotational energies can be expected

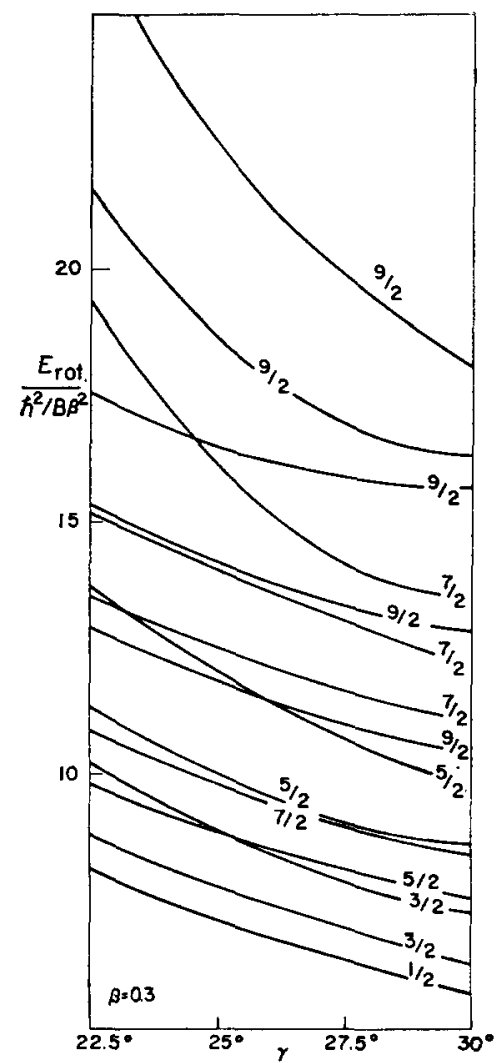

Fig. 4. Rotational energies for the particle state $63\left(\beta=0.3, \gamma>22.5^{\circ}\right)$. These are the natural continuation of the rotational levels of fig. 3. Near $\gamma \approx 20^{\circ}$, particle states 63 and 77 are nearly degenerate. For $\gamma>22.5^{\circ}$ the natural continuation of those for state 77 at smaller angles $\gamma$.

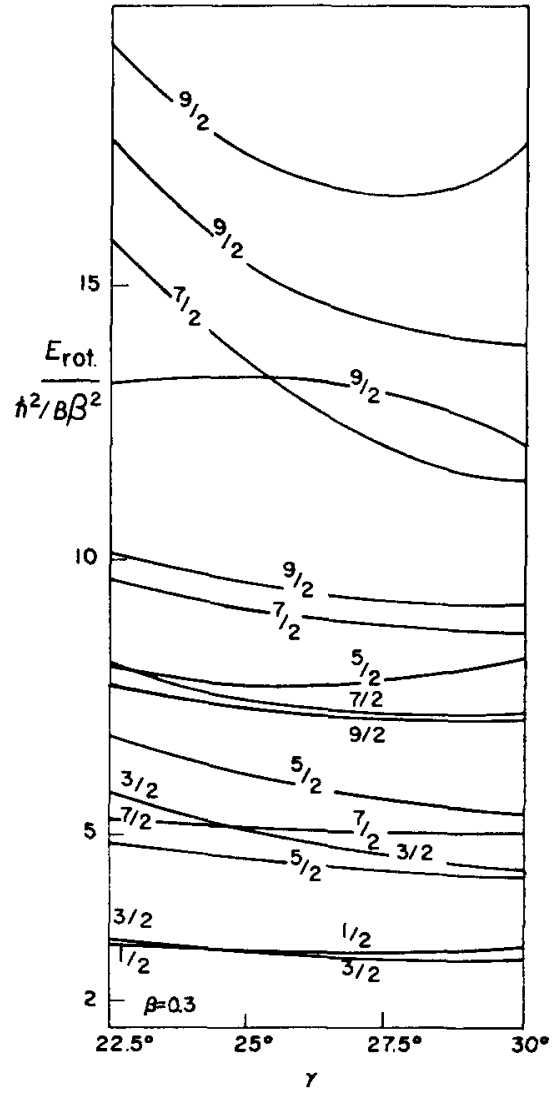

Fig. 5. Rotational energies for the particle state $77\left(\beta=0.3, \gamma>22.5^{\circ}\right)$.

to be small compared with the difference in particle energies. Now, however, particle state 63, the lower of the two, has a wave function whose character is very similar to that of the state $\mathbf{7 7}$ at smaller angles $\gamma$. The rotational energies based on particle level 63 should therefore form the natural continuation of 
the rotational energies of fig. 3. They are shown in fig. 4 for values of $\gamma$ between $22.5^{\circ}$ and $30^{\circ}$. The rotational energies based on particle level 77 for the same range of $\gamma$ are shown in fig. 5 .

Among the isotopes of Ir and $\mathrm{Au}$, which seem to have similar low energy spectra, the experimental situation seems to be most favourable for the isotope ${ }_{77} \mathrm{Ir}^{191}$. The experimentally observed low-lying states of $\operatorname{Ir}^{191}$ are shown in the

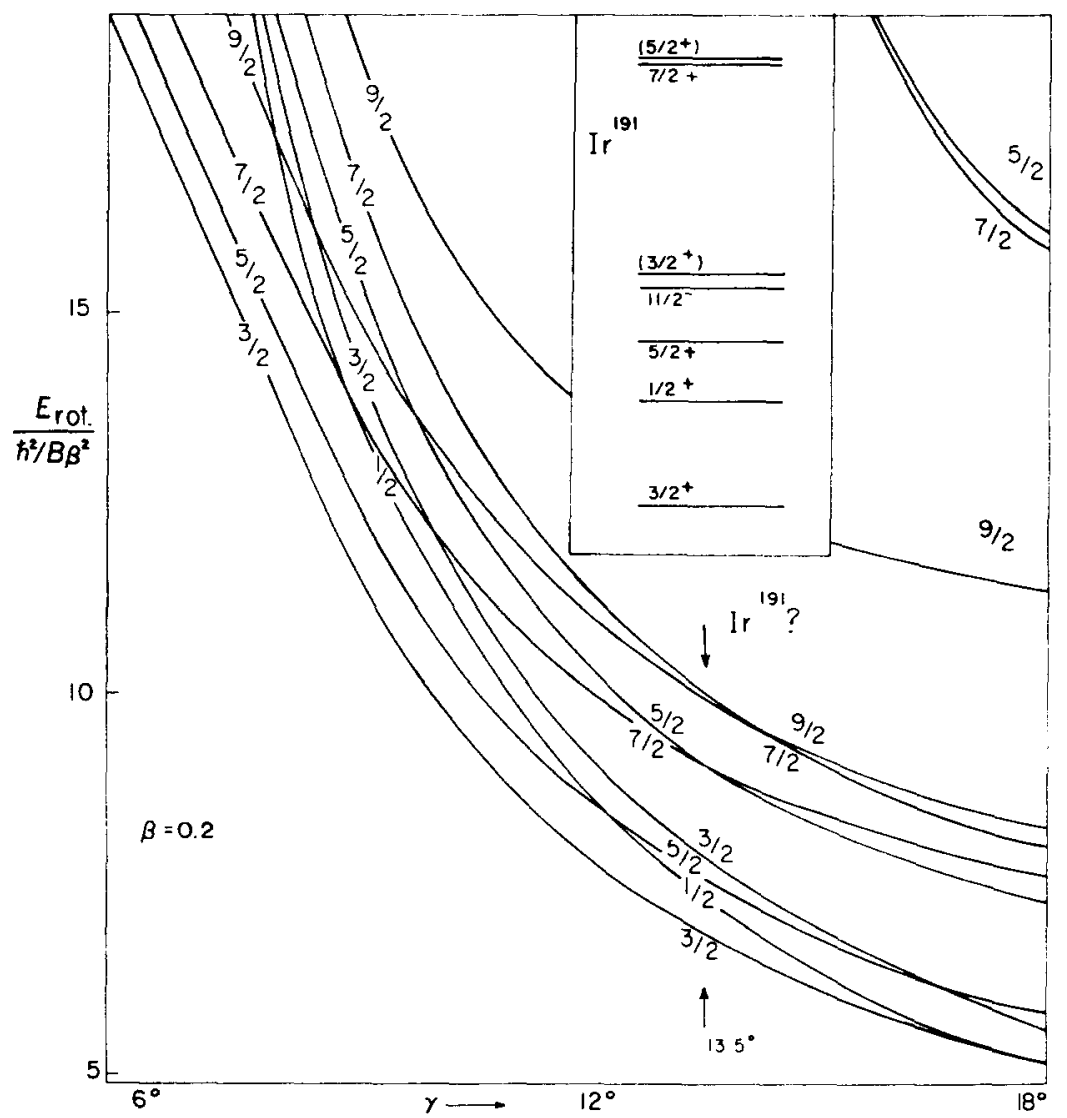

Fig. 6. Rotational energies for the particle state $79(\beta=0.2)$. As $\gamma$ approaches $0^{\circ}$, particle state 79 goes over to the axially symmetric [402] $\Omega=\frac{3}{2}+$ particle state. The insert shows the experimentally observed low-lying energy levels of ${ }_{77} \mathrm{Ir}^{101}$. Note that the order of the rotational levels for $\gamma=13.5^{\circ}(\beta=0.2)$ is in agreement with the experimentally observed + parity states in $\operatorname{Ir}^{191}$

insert of fig. 6. According to the axially symmetric model the low-lying positive parity states might be explained in terms of two rotational bands; the ground state band with $K=\frac{3}{2}$ based on the [402] $\Omega=\frac{3}{2}+$ Nilsson level, and a nearby band with $K=\frac{1}{2}$ based on the [400] $\Omega=\frac{1}{2}+$ Nilsson level. Because of the proximity of the two states the $K=\frac{1}{2}$ and $\frac{3}{2}$ bands would be coupled through the rotation-particle coupling (RPC) term ${ }^{13}$ ) in the rotational 
Hamiltonian. According to the asymmetric model, however, the positive parity particle levels $\mathbf{8 1}$ and $\mathbf{7 9}$ are split relatively far apart even for small asymmetry. (The limiting $\Omega= \pm \frac{1}{2}$ and $\Omega \mp \frac{3}{2}$ axially symmetric particle levels have very nearly the same energy and are connected by an off-diagonal matrix

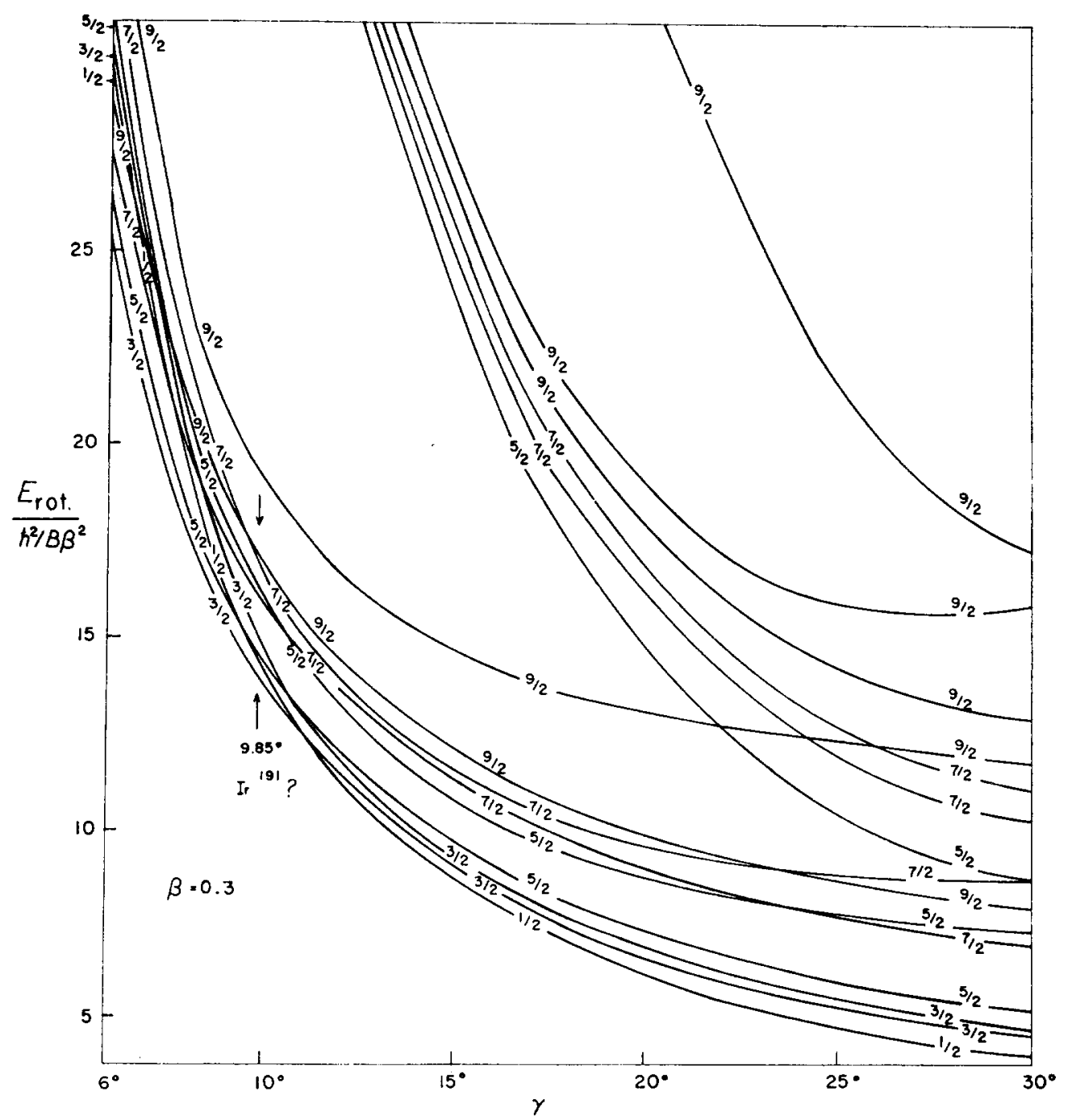

Fig. 7. Rotational energies for the particle state $79(\beta=0.3)$.

element when $\gamma \neq 0^{\circ}$ ). In the asymmetric model therefore the two particle states are far enough apart so that the observed rotational states should be based on a single particle state. Fig. 6 shows the rotational energies for particle level 79 as a function of $\gamma$ for the deformation $\beta=0.2$. Fig. 7 shows the rotation- 
al energies for the same particle level with $\beta=0.3$. The low-lying levels have the appearance of two overlapping rotational band systems, one a $\frac{3}{2}+, \frac{5}{2}+$, $\frac{7}{2}+, \ldots$ sequence, the other a $\frac{1}{2}+, \frac{3}{2}+, \frac{5}{2}+, \ldots$ sequence. (The zero point rotational energies are very large for $\gamma$ between $2^{\circ}$ and $6^{\circ}$ since the strong $\Omega=\frac{1}{2}, \frac{3}{2}$ mixing of the particle wave function gives a relatively large $K_{\mathrm{R}} \approx 2$ admixture for small values of $\gamma$ so that the $\hbar^{2}\left\langle K_{R}^{2}\right\rangle / 8 B \beta^{2} \sin ^{2} \gamma$ term in the rotational Hamiltonian gives a large energy contribution to all rotational levels.) For $\beta=0.2, \gamma=13.50^{\circ}$, and for $\beta=0.3, \gamma=9.85^{\circ}$ the observed levels of $\operatorname{Ir}^{191}$ are reproduced quite well. For intermediate values of $\beta$ there is always a value of $\gamma$ for which the predicted and observed energy spectra are in relatively good agreement.

TABLE 3

Rotational energies for $\operatorname{Ir}^{101}$

\begin{tabular}{|c|c|c|c|c|c|}
\hline & \multirow{3}{*}{ Experimental } & \multicolumn{4}{|c|}{ Theoretical } \\
\hline & & \multicolumn{2}{|c|}{ Asymmetric model } & \multicolumn{2}{|c|}{ Symmetric model with RPC } \\
\hline & & $\begin{array}{l}\beta=0.3 \\
\gamma=9.85^{\circ}\end{array}$ & $\begin{array}{l}\beta=0.2 \\
\left.\gamma=13.50^{\circ} \mathrm{b}\right)\end{array}$ & $\left.\beta=0.3^{\mathrm{c}}\right)$ & $\left.\beta=0.2^{\mathrm{d}}\right)$ \\
\hline $8+$ & $351 \mathrm{keV}$ & $377 \mathrm{keV}$ & $418 \mathrm{keV}$ & $305 \mathrm{keV}$ & $316 \mathrm{keV}$ \\
\hline$\frac{2}{2}+$ & 348 & 374 & 415 & 324 & 325 \\
\hline$\frac{3}{2}+$ & 178 & 173 & 178 & 208 & 206 \\
\hline$\frac{2}{2}+$ & 129 & (129) & (129) & (129) & $(129)$ \\
\hline $\begin{array}{l}2 \\
\frac{1}{2}+ \\
2\end{array}$ & 83 & 87 & 86 & (83) & (83) \\
\hline$\frac{2}{2}+$ & 0 & 0 & 0 & 0 & 0 \\
\hline
\end{tabular}

a) $\hbar^{2} / 6 B \beta^{2}=29.6 \mathrm{keV}$.

b) $\hbar^{2} / 6 B \beta^{2}=30.8 \mathrm{keV}$.

c) $\hbar^{2} / 6 B \beta^{2}=28.4 \mathrm{keV}, a=0.415, A=0.670, \Delta E=174.5 \mathrm{keV}$.

d) $\hbar^{2} / 6 B \beta^{2}=28.8 \mathrm{keV}, a=0.348, A=0.741, \Delta E=172.5 \mathrm{keV}$.

In these footnotes, $a$ is the computed decoupling parameter, $A$ the computed RPC parameter, $\Delta E$ is defined by $\Delta E=E\left([400]^{\frac{1}{2}}+\right)-E\left([402]^{\frac{3}{2}}+\right)$. The quantities $\hbar^{2} / 6 B \beta^{2}$ and $\Delta E$ are chosen empirically.

The theoretically predicted energies are shown in table $\mathbf{3}$ on the basis of both the asymmetric and the symmetric models. In the asymmetric model the rotational constant, $\hbar^{2} / B \beta^{2}$, was determined empirically to fit the $129 \mathrm{keV}$ level. The predicted energies for the upper $\frac{5}{2}+$ and $\frac{7}{2}+$ states are somewhat too high but this is perhaps not disturbing since vibration-rotation interactions have not been taken into account. In the symmetric model the rotational energies were computed by diagonalizing the symmetric rotator Hamiltonian with RPC term. The values of the decoupling parameter $a$ for the $\Omega=\frac{1}{2}$ state and the coupling parameter $A^{13}$ ), were computed from Nilsson wave functions and are shown in table 3 . In the symmetric case the determination of the rotational energies involved two empirical constants, the energy difference between the $\Omega=\frac{1}{2}$ and $\Omega=\frac{3}{2}$ particle states and a rotational constant $\hbar^{2} / 2 \mathscr{I}=\hbar^{2} / 6 B \beta^{2}$ 
which was assumed to have the same value for both bands. Although the predicted energy values may be somewhat better on the basis of asymmetric theory (if it is borne in mind that vibration-rotation interactions are apt to depress the higher rotational states somewhat), no preference can be given to either model. The rotational wave functions are very similar for both models since the overlapping $\frac{3}{2}+, \frac{5}{2}+, \frac{7}{2}+\ldots$ and $\frac{1}{2}+, \frac{3}{2}+, \frac{5}{2}+\ldots$ sequences in the asymmetric model are predominantly $K=\frac{3}{2}$ and $K=\frac{1}{2}$, respectively. The ground state which is predominantly $K=\frac{\mathbf{3}}{\mathbf{2}}$ has a $K=\frac{1}{2}$ admixture with amplitudes $C_{\frac{1}{2}}$ between -0.1 and -0.2 in the case of both models. As a result it is again difficult to distinguish between the two models as far as predicted values of the electromagnetic moments and transition probabilities are concerned. Table 4 shows that neither model is very successful in predicting

TABLE 4

Electromagnetic moments and transition probabilities for $\mathrm{Ir}^{191}$

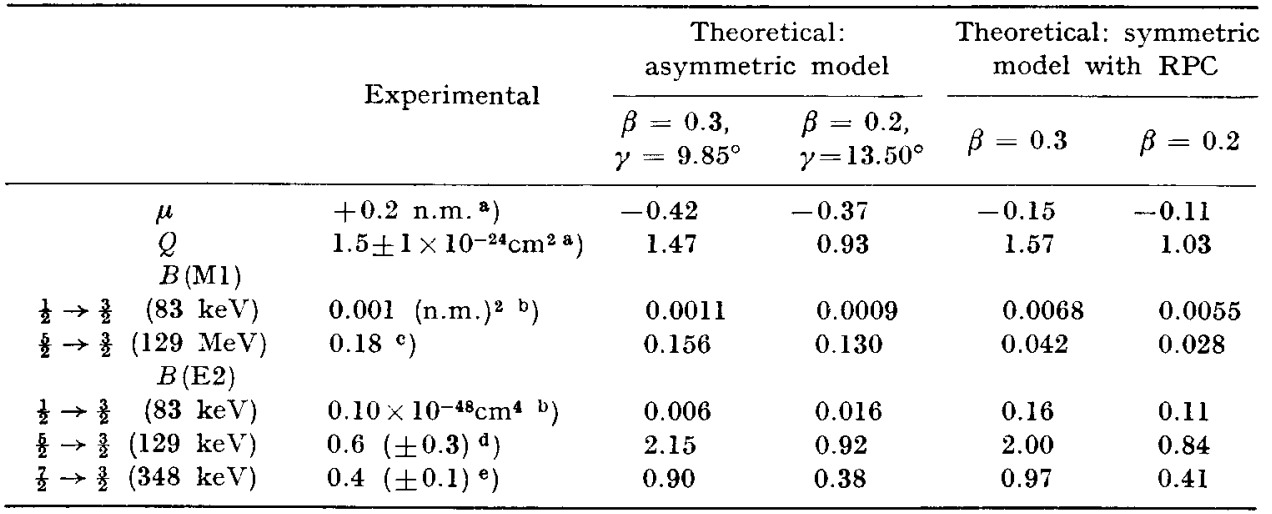

a) W. v. Siemens, Ann. Physik 13 (1953) 136; K. Murakawa and S. Suwa, Phys. Rev. 87 (1952) 1048

b) A. W. Sunyar, Phys. Rev. 98 (1955) 653

c) Using a meanlife $1.4 \times 10^{-10} \mathrm{sec}$ (R, L. Mössbauer, Z. Naturforschung 14a (1959) 211)

d) T. Huus, J. Bjerregaard, B. Elbek, Dan. Vid. Selsk. Mat. Fys. Medd. 30, No. 17 (1956); E. M. Bernstein and H. W. Lewis, Phys. Rev. 105 (1957) 1524

e) R. H. Davis, A. S. Divatia, D. A. Lind, R. D. Moffat, Phys. Rev. 101 (1956) 753

the experimentally observed magnetic moment and in giving consistent predictions for the observed Ml and E2 transition probabilities. (Free nucleon $g_{s}$ values are used in all the magnetic moment calculations, and $g_{\mathrm{R}}$ is set equal to $Z / A$. A value of $R_{\mathbf{0}}=1.2 \cdot A^{\frac{1}{3}} \mathrm{fm}$ is used for all electric quadrupole calculations). The $\operatorname{Ir}^{191}$ calculations seem to indicate that it may always be difficult to distinguish between a symmetric and an asymmetric model when the asymmetry is relatively small.

\subsection{ODD-NEUTRON NUCLEI}

Figs. 8 and 9 show the single particle energy levels for odd-neutron nuclei in the region $82<N<126$ for asymmetries of $15^{\circ}$ and $30^{\circ}$, respectively. For 
the specific deformations $\beta=0.3$ and $\beta=-0.2$ the spin values of the lowest rotational levels based on each particle state are also listed in table $\mathbf{5}$ (starting with the ground state at the left), in order to give at least some indication of the types of rotational sequences to be expected. Particle levels 121 to 115, and perhaps level 99 may be the pertinent levels for odd parity states of nuclei with $A$ around 190.

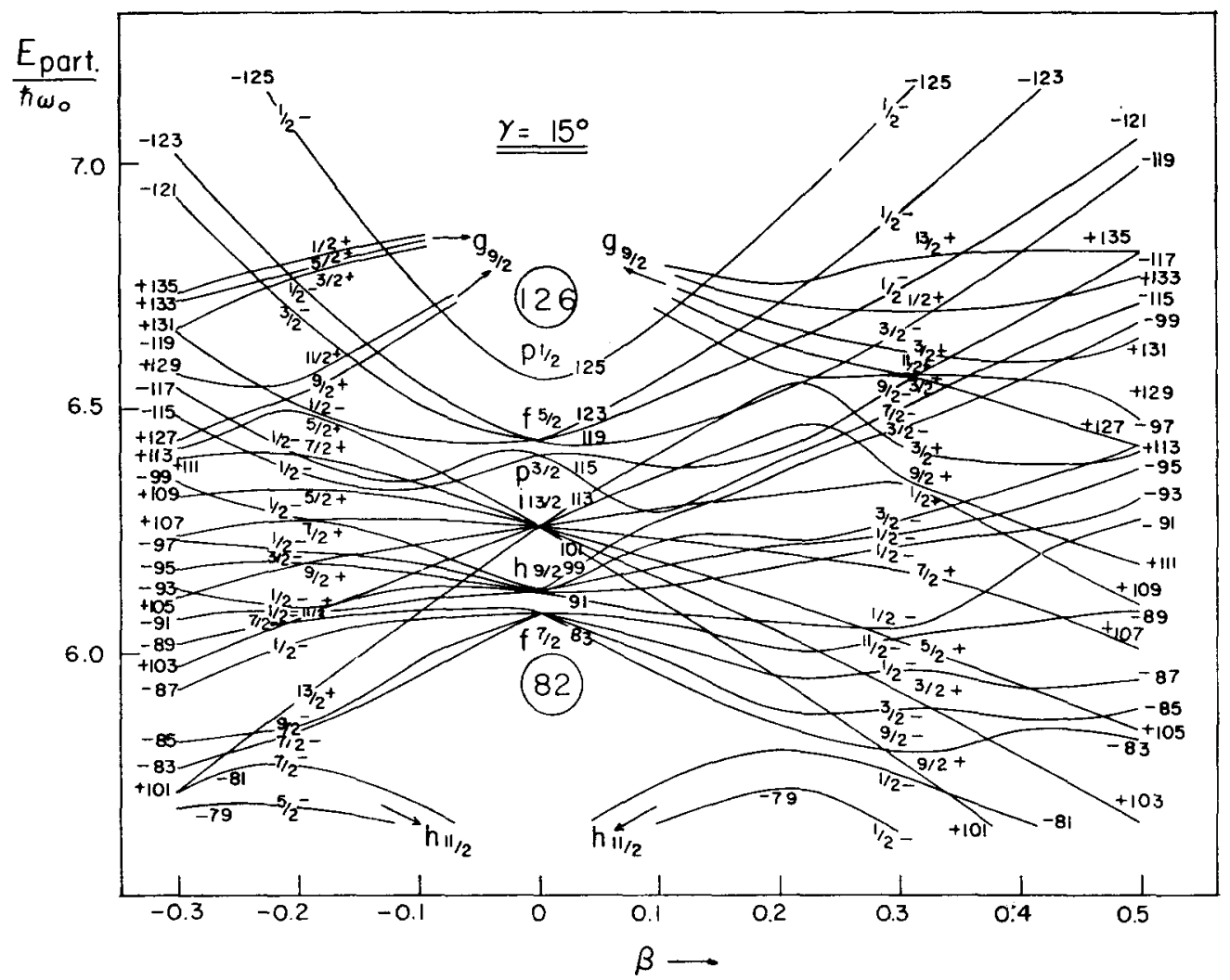

Fig. 8. Single-particle levels for odd-neutron nuclei in the region $82<N<126\left(\gamma=15^{\circ}\right)$. The parameters $C(\kappa)$ and $D(\mu)$ for these levels have been chosen to have the values recommended by Mottelson and Nilsson ${ }^{\circ}$ ). The calculated wave functions correspond to the parameters $C=-0.1$ $(\kappa=0.05)$ and $D=-0.0225(\mu=0.45)$ for both the - parity $N=5$ and + parity $N=6$ levels. The levels are labelled by their parity and by an ordering number which would be the number of the odd neutron if the single-particle levels, at small deformation $\beta$, were filled in order. The single particle levels are also labelled with the spin and parity of the lowest rotational level (ground state) for the specific deformations $\beta=0.3$ and $\beta=-0.2$.

The nucleus ${ }_{78} \mathrm{Pt}^{195}$ is of particular interest since its energy spectrum is seemingly fit by asymmetric rotator theory. The experimentally observed energy levels are shown on the left hand side of fig. 10. The levels have been studied through Coulomb excitation ${ }^{14}$ ), the decay of the metastable $\frac{13}{2}+$ level and the positon decay of $\mathrm{Au}^{195}$ to the low-lying states. The most striking feature 
of the level scheme is that both the upper and lower $\frac{\mathbf{3}}{2}-, \frac{5}{2}-$ doublets show large electric quadrupole transition probabilities to the ground state, the E2 strengths being 10 to 30 times the single particle estimates ${ }^{14}$ ). In a rough way each doublet seems to correspond to a state with a rotational (or vibrational) angular momentum of 2 units. Fig. 10 shows that there are actually three different particle energy levels all with theoretically predicted rotational spectra which seem to reproduce the experimentally observed level scheme. All three particle states fall in the region appropriate for the $117^{\text {th }}$ neutron in $\mathrm{Pt}^{195}$, and

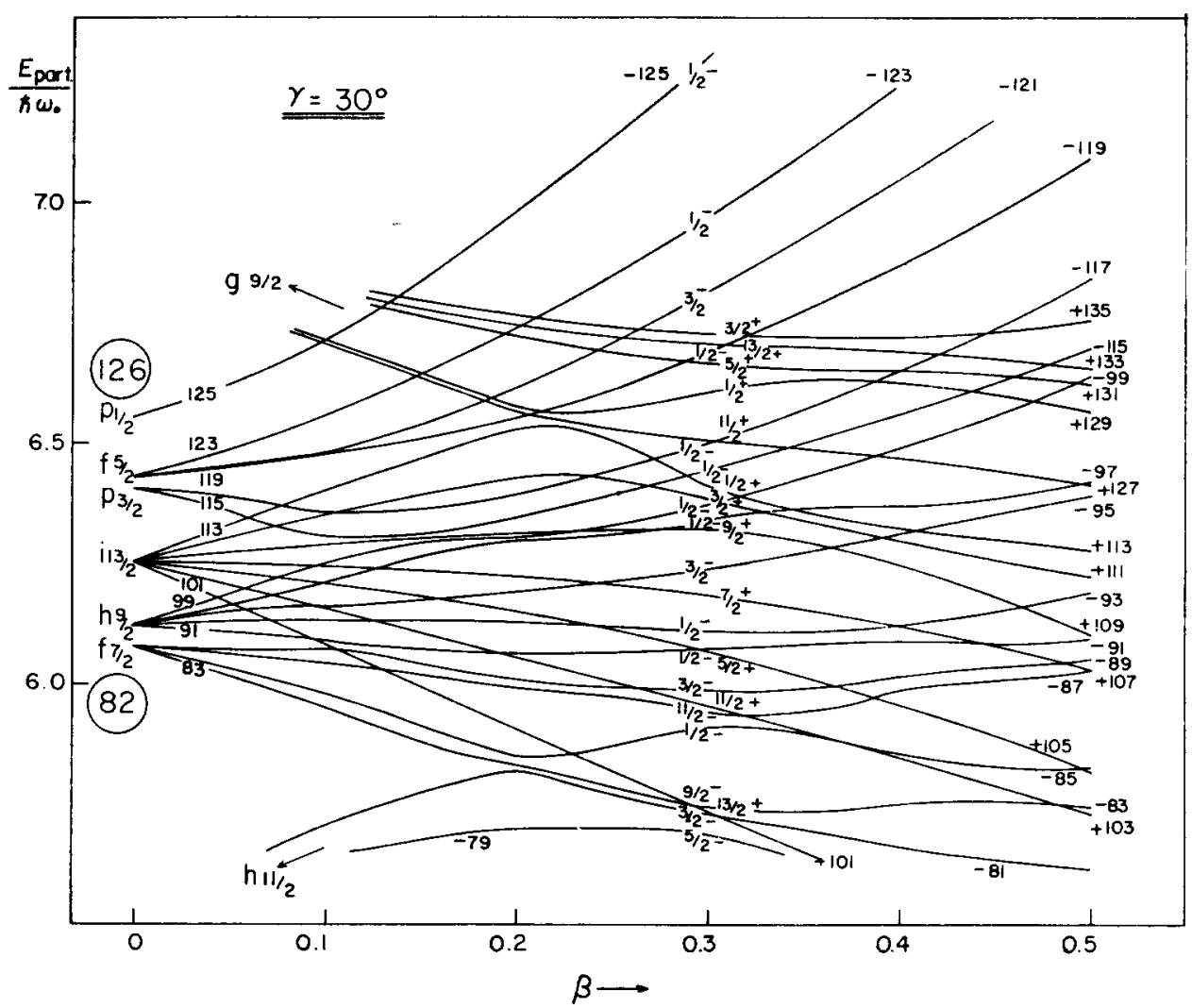

Fig. 9. Single-particle levels for odd-neutron nuclei in the region $82<N<126\left(\gamma=30^{\circ}\right)$. See caption for fig. 8 .

all three seem to imply large asymmetry. Moreover for any value of $\beta$ within the limits to be expected in this region of the periodic table there seems to be a value of $\gamma$ for which the observed level scheme is reproduced at least tolerably well. For $\beta=0.1, \gamma \approx 30^{\circ}$, similarly for $\beta=0.2, \gamma \approx 23^{\circ}$, however, particle levels 119 and 121 have nearly the same energy so that rotational energy calculations based on a single particle state may not be valid. For the values of $\beta$ and $\gamma$ used in fig. 10, the energy separations between single particle states 
TABLE 5

Order of low-lying rotational levels for odd-neutron nuclei ${ }^{8}$ )

\begin{tabular}{|c|c|c|c|c|c|c|c|c|c|c|c|c|c|c|}
\hline \multicolumn{2}{|r|}{$\begin{array}{r}\text { Part. } \\
\text { state }\end{array}$} & $\frac{\text { Par. }}{-}$ & $\begin{array}{c}\text { Gr. st. } \\
\text { spin }\end{array}$ & \multicolumn{4}{|c|}{$\begin{array}{l}\text { Spins of lowest } \\
\text { rotational states }\end{array}$} & $\frac{\begin{array}{c}\text { Part. } \\
\text { state }\end{array}}{109}$ & $\frac{\text { Par. }}{+}$ & $\begin{array}{c}\begin{array}{c}\text { Gr. st. } \\
\text { spin }\end{array} \\
\frac{1}{\frac{1}{2}}\end{array}$ & \multicolumn{4}{|c|}{$\begin{array}{l}\text { Spins of lowest } \\
\text { rotational states }\end{array}$} \\
\hline $\begin{array}{l}\stackrel{0}{0} \\
\|\end{array}$ & $\begin{array}{r}125 \\
123 \\
135 \\
121 \\
133 \\
119 \\
131 \\
129 \\
127 \\
117 \\
115 \\
99 \\
113 \\
111 \\
\end{array}$ & $\begin{array}{l}- \\
- \\
+ \\
- \\
+ \\
- \\
+ \\
+ \\
+ \\
- \\
- \\
- \\
+ \\
+\end{array}$ & $\begin{array}{c}\frac{1}{2} \\
\frac{1}{2} \\
\frac{13}{2} \\
\frac{1}{2} \\
\frac{1}{2} \\
\frac{3}{2} \\
\frac{3}{2} \\
\frac{3}{2} \\
\frac{3}{2} \\
\frac{9}{2} \\
\frac{7}{2} \\
\frac{3}{2} \\
\frac{3}{2} \\
\frac{9}{2} \\
\end{array}$ & $\begin{array}{ll}\frac{3}{2} & \\
\frac{3}{2} & \\
\frac{9}{2} & 1 \\
\frac{3}{2} & \\
\frac{5}{2} & \\
\frac{1}{2} & \\
\frac{1}{2} & \\
\frac{13}{2} & \\
\frac{5}{2} & \\
\frac{8}{2} & 1 \\
\frac{9}{2} & \\
\frac{1}{2} \\
\frac{1}{2} \\
\frac{11}{2} \\
\end{array}$ & $\begin{array}{ll}\frac{5}{2} & \frac{7}{2} \\
\frac{5}{2} & \frac{7}{2} \\
\frac{11}{2} & \frac{13}{2} \\
\frac{5}{2} & \frac{7}{2} \\
\frac{3}{2} & \frac{5}{2} \\
\frac{3}{2} & \frac{5}{2} \\
\frac{5}{2} & \frac{3}{2} \\
\frac{7}{2} & \frac{8}{2} \\
\frac{7}{2} & \frac{1}{2} \\
\frac{11}{2} & \frac{7}{2} \\
\frac{3}{2} & \frac{5}{2} \\
\frac{3}{2} & \frac{5}{2} \\
\frac{5}{2} & \frac{7}{2} \\
\frac{13}{2} & \frac{5}{4} \\
\end{array}$ & 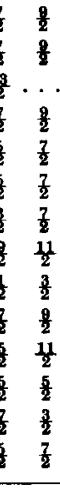 & $\begin{array}{l}\frac{11}{2} \ldots \\
\frac{11}{2} \ldots \\
\begin{array}{l} \\
\frac{3}{2}\end{array} \ldots \\
\frac{9}{2} \ldots \\
\frac{1}{2} \ldots \\
\frac{5}{2} \ldots \\
\frac{13}{2} \ldots \\
\frac{9}{2} \ldots \\
\frac{11}{2} \ldots \\
\frac{7}{2} \ldots \\
\frac{7}{2} \ldots \\
\frac{9}{2} \ldots \\
\frac{9}{2} \ldots \\
\end{array}$ & $\begin{array}{r}109 \\
97 \\
95 \\
93 \\
107 \\
91 \\
89 \\
105 \\
87 \\
103 \\
85 \\
83 \\
101\end{array}$ & $\begin{array}{l}+ \\
- \\
- \\
- \\
+ \\
- \\
- \\
+ \\
+ \\
+ \\
- \\
+\end{array}$ & $\begin{array}{l}\frac{1}{2} \\
\frac{3}{2} \\
\frac{1}{2} \\
\frac{1}{2} \\
\frac{7}{2} \\
\frac{7}{2} \\
\frac{1}{2} \\
\frac{1}{2} \\
\frac{5}{2} \\
\frac{1}{2} \\
\frac{3}{2} \\
\frac{3}{2} \\
\frac{9}{2} \\
\frac{9}{2}\end{array}$ & $\begin{array}{ll}\frac{3}{2} & \\
\frac{5}{2} & \\
\frac{3}{2} & \\
\frac{3}{2} & \\
\frac{9}{2} & 1 \\
\frac{3}{2} & \\
\frac{2}{2} & \\
\frac{7}{2} & \\
\frac{7}{2} & \\
\frac{3}{2} & \\
\frac{7}{2} & \\
\frac{5}{2} & \\
\frac{11}{2} & \\
\frac{13}{2}\end{array}$ & $\begin{array}{ll}\frac{5}{2} & \frac{7}{2} \\
\frac{7}{2} & \frac{7}{2} \\
\frac{5}{2} & \frac{7}{8} \\
\frac{8}{8} & \frac{8}{2} \\
\frac{5}{2} & \frac{13}{2} \\
\frac{5}{2} & \frac{7}{2} \\
\frac{9}{2} & \frac{3}{2} \\
\frac{9}{2} & \frac{11}{2} \\
\frac{9}{2} & \frac{1}{2} \\
\frac{5}{2} & \frac{3}{2} \\
\frac{5}{2} & \frac{8}{2} \\
\frac{1}{2} & \frac{3}{2} \\
\frac{5}{2} & \frac{7}{2} \\
\frac{5}{2} & \frac{1}{2}\end{array}$ & $\begin{array}{c} \\
\frac{9}{2} \\
\frac{9}{2} \\
\frac{9}{2} \\
\frac{7}{2} \\
\frac{7}{2} \\
\frac{3}{2} \\
\frac{3}{2} \\
\frac{5}{2} \\
\frac{1}{2} \\
\frac{1}{2} \\
\frac{11}{2} \\
\frac{5}{2} \\
\frac{3}{2} \\
\frac{11}{2} \\
\frac{3}{2} \\
\frac{7}{2} \\
\frac{2}{2} \\
\frac{8}{2} \\
\frac{7}{2}\end{array}$ & $\begin{array}{l}\frac{12}{2} \ldots \\
\frac{8}{2}\end{array} \ldots$ \\
\hline $\begin{array}{l}01 \\
0 \\
1 \\
11 \\
0\end{array}$ & $\begin{array}{l}125 \\
135 \\
133 \\
131 \\
123 \\
121 \\
129 \\
127 \\
119 \\
113 \\
111 \\
117 \\
115 \\
109 \\
\end{array}$ & $\begin{array}{l}- \\
+ \\
+ \\
+ \\
- \\
- \\
+ \\
+ \\
\overline{+} \\
+ \\
- \\
+ \\
+\end{array}$ & $\begin{array}{l}\frac{7}{2} \\
\frac{1}{2} \\
\frac{5}{2} \\
\frac{3}{2} \\
\frac{1}{2} \\
\frac{3}{2} \\
\frac{1}{2} \\
\frac{8}{2} \\
\frac{1}{2} \\
\frac{5}{2} \\
\frac{7}{2} \\
\frac{1}{2} \\
\frac{1}{2} \\
\frac{5}{2} \\
\end{array}$ & $\begin{array}{l}\frac{3}{2} \\
\frac{5}{2} \\
\frac{7}{2} \\
\frac{5}{2} \\
\frac{3}{2} \\
\frac{5}{2} \\
\frac{13}{2} \\
\frac{11}{2} \\
\frac{3}{2} \\
\frac{1}{2} \\
\frac{11}{2} \\
\frac{3}{2} \\
\frac{3}{2} \\
\frac{1}{2} \\
\end{array}$ & $\begin{array}{ll}\frac{5}{2} & \frac{7}{2} \\
\frac{3}{2} & \frac{7}{2} \\
\frac{8}{2} & \frac{1}{2} \\
\frac{1}{2} & \frac{7}{2} \\
\frac{5}{2} & \frac{3}{2} \\
\frac{1}{2} & \frac{3}{2} \\
\frac{7}{2} & \frac{8}{2} \\
\frac{6}{2} & \frac{7}{2} \\
\frac{5}{2} & \frac{7}{2} \\
\frac{11}{2} & \frac{7}{2} \\
\frac{3}{2} & \frac{5}{2} \\
\frac{6}{2} & \frac{7}{2} \\
\frac{5}{2} & \frac{6}{2} \\
\frac{9}{2} & \frac{1}{2} \\
\end{array}$ & $\begin{array}{ll}\frac{7}{2} & \frac{9}{2} \\
\frac{7}{2} & \frac{9}{2} \\
\frac{1}{2} & \frac{3}{2} \\
\frac{7}{2} & \frac{5}{2} \\
\frac{3}{2} & \frac{7}{2} \\
\frac{3}{2} & \frac{7}{2} \\
\frac{9}{2} & \frac{11}{2} \\
\frac{7}{2} & \frac{13}{2} \\
\frac{7}{2} & \frac{9}{2} \\
\frac{7}{2} & \frac{3}{2} \\
\frac{5}{2} & \frac{8}{2} \\
\frac{7}{2} & \frac{3}{2} \\
\frac{6}{2} & \frac{7}{2} \\
\frac{13}{2} & \frac{3}{2} \\
\end{array}$ & $\begin{array}{l}\frac{y}{2} \ldots \\
\frac{11}{2} \ldots \\
\frac{41}{2} \ldots \\
\frac{9}{2} \ldots \\
\frac{5}{2} \ldots \\
\frac{5}{2} \ldots \\
\frac{13}{2} \ldots \\
\frac{9}{2} \ldots \\
\frac{3}{2} \ldots \\
\frac{3}{2} \ldots \\
\frac{5}{2} \ldots \\
\frac{1}{2} \ldots \\
\frac{8}{2} \ldots \\
\frac{8}{2} \ldots \\
\frac{8}{2} \ldots\end{array}$ & $\begin{array}{r}99 \\
107 \\
97 \\
95 \\
105 \\
93 \\
91 \\
103 \\
89 \\
87 \\
101 \\
85 \\
83\end{array}$ & $\begin{array}{l}- \\
+ \\
- \\
- \\
+ \\
- \\
- \\
+ \\
- \\
- \\
+ \\
- \\
-\end{array}$ & $\begin{array}{l}\frac{1}{2} \\
\frac{7}{2} \\
\frac{1}{2} \\
\frac{3}{2} \\
\frac{8}{2} \\
\frac{1}{2} \\
\frac{1}{2} \\
\frac{1}{2} \\
\frac{7}{2} \\
\frac{7}{2} \\
\frac{1}{2} \\
\frac{13}{2} \\
\frac{9}{2} \\
\frac{7}{2}\end{array}$ & $\begin{array}{c}\frac{5}{2} \\
\frac{9}{2} \\
\frac{3}{2} \\
\frac{1}{2} \\
\frac{1}{2} \\
\frac{3}{2} \\
\frac{5}{2} \\
\frac{13}{2} \\
\frac{8}{2} \\
\frac{3}{2} \\
\frac{8}{2} \\
\frac{11}{2} \\
\frac{8}{2}\end{array}$ & $\begin{array}{ll}\frac{3}{2} & \frac{7}{2} \\
\frac{3}{2} & \frac{5}{2} \\
\frac{5}{2} & \frac{7}{2} \\
\frac{5}{2} & \frac{3}{2} \\
\frac{13}{2} & \frac{5}{2} \\
\frac{5}{2} & \frac{7}{2} \\
\frac{2}{2} & \frac{8}{2} \\
\frac{3}{2} & \frac{8}{2} \\
\frac{3}{2} & \frac{5}{2} \\
\frac{2}{2} & \frac{5}{2} \\
\frac{5}{2} & \frac{3}{2} \\
\frac{11}{2} & 1 \\
\frac{5}{2} & \\
\frac{4}{2} & \\
\frac{1}{2} & \end{array}$ & 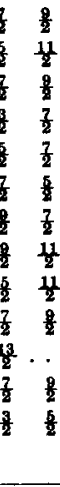 & 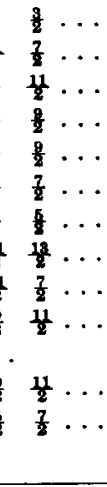 \\
\hline $\begin{array}{l}\mathfrak{n}_{0}^{\circ} \\
\| \\
0\end{array}$ & $\begin{array}{r}125 \\
123 \\
121 \\
135 \\
133 \\
119 \\
131 \\
129 \\
127 \\
117 \\
115 \\
113 \\
111 \\
99\end{array}$ & $\begin{array}{l}- \\
- \\
- \\
+ \\
+ \\
\overline{+} \\
+ \\
+ \\
+ \\
- \\
- \\
+ \\
+ \\
-\end{array}$ & $\begin{array}{l}\frac{3}{2} \\
\frac{3}{2} \\
\frac{13}{2} \\
\frac{1}{2} \\
\frac{5}{2} \\
\frac{1}{2} \\
\frac{1}{2} \\
\frac{1}{2} \\
\frac{1}{2} \\
\frac{1}{2} \\
\frac{3}{2} \\
\frac{1}{2}\end{array}$ & $\begin{array}{l}\frac{3}{2} \\
\frac{3}{2} \\
\frac{1}{2} \\
\frac{1}{2} \\
\frac{1}{2} \\
\frac{9}{2} \\
\frac{3}{2} \\
\frac{2}{2} \\
\frac{3}{2} \\
\frac{3}{2} \\
\frac{7}{2} \\
\frac{3}{2} \\
\frac{3}{2} \\
\frac{3}{2} \\
\frac{7}{2} \\
\frac{5}{2} \\
\end{array}$ & $\begin{array}{ll}\frac{5}{2} & \frac{3}{2} \\
\frac{3}{2} & \frac{8}{2} \\
\frac{3}{2} & \frac{5}{2} \\
\frac{5}{2} & \frac{7}{2} \\
\frac{11}{2} & \frac{5}{2} \\
\frac{5}{2} & \frac{3}{2} \\
\frac{3}{2} & \frac{7}{2} \\
\frac{3}{2} & \frac{5}{2} \\
\frac{13}{2} & \frac{9}{2} \\
\frac{3}{2} & \frac{5}{2} \\
\frac{5}{2} & \frac{7}{2} \\
\frac{5}{2} & \frac{5}{2} \\
\frac{5}{2} & \frac{1}{2} \\
\frac{3}{2} & \frac{5}{2}\end{array}$ & $\begin{array}{ll}\frac{3}{2} & \frac{5}{2} \\
\frac{5}{2} & \frac{5}{2} \\
\frac{5}{2} & \frac{7}{2} \\
\frac{7}{2} & \frac{8}{2} \\
\frac{5}{2} & \frac{13}{2} \\
\frac{3}{2} & \frac{5}{2} \\
\frac{7}{2} & \frac{9}{2} \\
\frac{5}{2} & \frac{7}{2} \\
\frac{9}{2} & \frac{3}{2} \\
\frac{5}{2} & \frac{7}{2} \\
\frac{7}{2} & \frac{5}{2} \\
\frac{5}{2} & \frac{3}{2} \\
\frac{1}{2} & \frac{7}{2} \\
\frac{5}{2} & \frac{3}{2}\end{array}$ & $\begin{array}{l}\frac{7}{2} \ldots \\
\frac{7}{8} \ldots \\
\frac{8}{2} \ldots \\
\frac{9}{2} \ldots \\
\frac{7}{2} \ldots \\
\frac{7}{2} \ldots \\
\frac{5}{8} \ldots \\
\frac{5}{2} \ldots \\
\frac{5}{2} \ldots \\
\frac{11}{2} \ldots \\
\frac{5}{2} \ldots \\
\frac{3}{2} \ldots \\
\frac{7}{2} \ldots \\
\frac{9}{2} \ldots \\
\frac{7}{2} \ldots\end{array}$ & $\begin{array}{r}97 \\
109 \\
95 \\
107 \\
93 \\
91 \\
105 \\
89 \\
103 \\
87 \\
85 \\
83 \\
101\end{array}$ & $\begin{array}{l}- \\
+ \\
\overline{+} \\
- \\
- \\
+ \\
- \\
+ \\
- \\
- \\
- \\
+\end{array}$ & $\begin{array}{l}\frac{1}{2} \\
\frac{8}{2} \\
\frac{3}{2} \\
\frac{7}{2} \\
\frac{1}{2} \\
\frac{1}{2} \\
\frac{5}{2} \\
\frac{3}{2} \\
\frac{41}{2} \\
\frac{1}{2} \\
\frac{1}{2} \\
\frac{9}{2} \\
\frac{13}{2}\end{array}$ & $\begin{array}{l}\frac{3}{2} \\
\frac{5}{2} \\
\frac{1}{2} \\
\frac{3}{2} \\
\frac{3}{2} \\
\frac{3}{2} \\
\frac{9}{2} \\
\frac{5}{2} \\
\frac{7}{2} \\
\frac{9}{2} \\
\frac{3}{2} \\
\frac{5}{2} \\
\frac{9}{2}\end{array}$ & 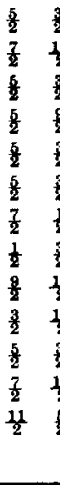 & $\begin{array}{ll}\frac{3}{2} & \frac{5}{2} \\
\frac{11}{2} & \frac{13}{2} \\
\frac{3}{2} & \frac{7}{2} \\
\frac{9}{2} & \frac{11}{2} \\
\frac{3}{2} & \frac{7}{2} \\
\frac{3}{2} & \frac{7}{2} \\
\frac{1}{2} & \frac{5}{2} \\
\frac{3}{2} & \frac{7}{2} \\
\frac{13}{2} & \frac{3}{2} \\
\frac{11}{2} & \frac{5}{2} \\
\frac{3}{2} & \frac{5}{2} \\
\frac{11}{2} & \frac{8}{2} \\
\frac{6}{2} & \frac{1}{2}\end{array}$ & $\left.\begin{array}{l}\frac{7}{2} \\
\frac{1}{8}\end{array}\right]$ \\
\hline
\end{tabular}

a) Levels with $I \leqq \frac{13}{2}$ are listed for the positive parity states, $I \leqq \frac{11}{2}$ for the negative parity states.

119 and 121 seem to be large enough for at least the approximate validity of the simple model. The theoretically predicted $\frac{7}{2}-$ and $\frac{9}{2}-$ levels which fall above the $\frac{13}{2}+$ metastable state are dashed since they would not have been 
experimentally observed. A third $\frac{\mathbf{5}}{\mathbf{2}}-$ state (above $259 \mathrm{keV}$ ), however, would be in agreement with experiment only if the E2 transition probability from the ground state is small.

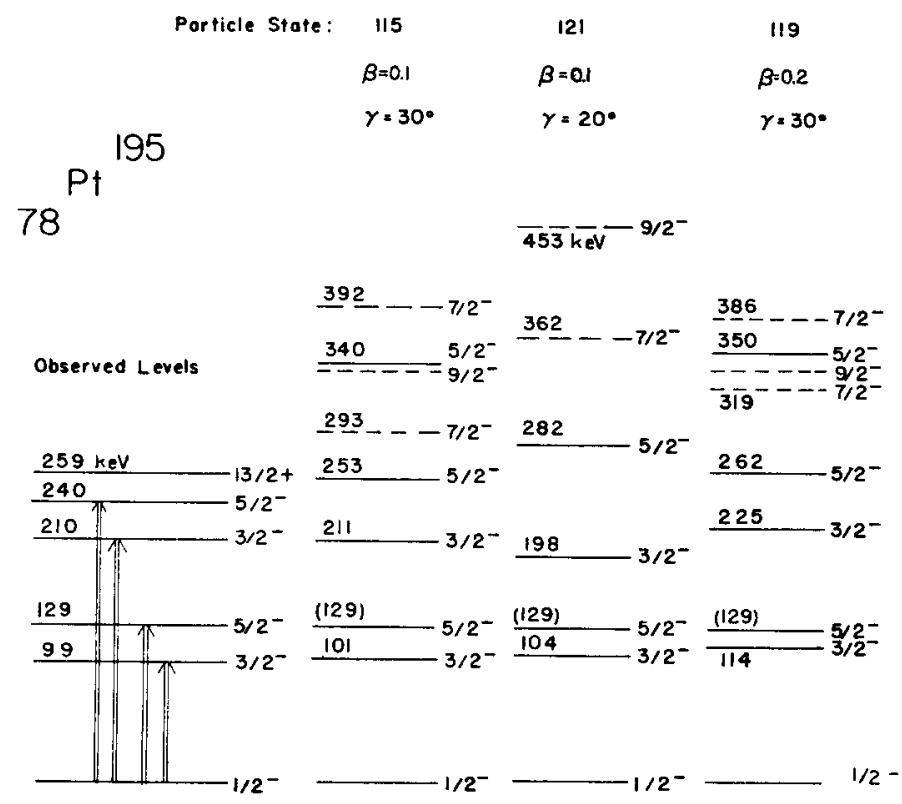

Fig. 10. Energy levels for ${ }_{78} \mathrm{Pt}^{195}$. The experimentally observed levels are shown on the left. Level schemes predicted on asymmetric rotator theory are shown for three different negative parity particle states in the region appropriate for the $117^{\text {th }}$ neutron. Predicted levels with $I=\frac{7}{2}$ and $\frac{8}{2}$ above $259 \mathrm{keV}$ have been drawn with dashed lines since they would probably not have been experimentally observed. The predicted third $I=\frac{6}{2}$ levels (above $259 \mathrm{keV}$ ) are in agreement with the observed facts only if they are connected to the ground state through very small E2 transition probabilities. The rotational constant $\hbar^{2} / B \beta^{2}$ has been chosen to fit the 129 $\mathrm{keV}$ level in each case. Values of $\hbar^{2} / B \beta^{2}$ of $80 \mathrm{keV}, 100 \mathrm{keV}$ and $82 \mathrm{keV}$ were used for particle states 115,121 and 119.

TABLE 6

Magnetic moment and M1 transition probabilities in $\mathrm{Pt}^{195}$

\begin{tabular}{lcccc}
\hline & Expt. & $\begin{array}{c}\text { State } 115 \\
\beta=0.1 \gamma=30^{\circ}\end{array}$ & $\begin{array}{c}\text { State } 121 \\
\beta=0.1 \gamma=20^{\circ}\end{array}$ & $\begin{array}{c}\text { State } 119 \\
\beta=0.2 \gamma=30^{\circ}\end{array}$ \\
\hline \multicolumn{1}{c}{$\mu$ (n.m.) } & 0.600 & 0.689 & 0.726 & 0.667 \\
\hline$B(\mathrm{M} 1)(\mathrm{n} . \mathrm{m})^{2}$ & & & & \\
\hline$\frac{3}{2} \rightarrow \frac{1}{2}(210 \mathrm{keV})$ & 0.047 & 0.076 & 0.392 & 0.088 \\
$\frac{3}{2} \rightarrow \frac{1}{2}(99 \mathrm{keV})$ & $(\mathbf{0 . 0 4 4 )}$ & 0.072 & 0.017 & 0.112 \\
$\frac{5}{2} \rightarrow \frac{3}{2}(140 \mathrm{keV})$ & 0.0087 & 0.0056 & 0.242 & 0.014 \\
$\frac{5}{2} \rightarrow \frac{3}{2}(31 \mathrm{keV})$ & 0.071 & 0.024 & 0.306 & 0.165 \\
\hline
\end{tabular}

The experimentally observed and theoretically predicted values for the magnetic moment are shown in table 6 . These are determined mainly by the particle 
wave functions since an $I=\frac{1}{2}$ level is a pure $K=\frac{1}{2}$ level for all values of $\gamma$. The reduced widths for magnetic dipole transitions seem to rule out the second possibility; but considering the limitations of the model and perhaps experimental uncertainties, they seem to give fair agreement for the other two levels.

\section{TABLE 7}

E2 transition probabilities in $\mathrm{Pt}^{105}$

\begin{tabular}{|c|c|c|c|c|c|}
\hline & $\begin{array}{c}B(\mathrm{E} 2) \\
10^{-40} \mathrm{~cm}^{4}\end{array}$ & Expt. ${ }^{14}$ ) & $\begin{array}{c}\text { State } 115 \\
\beta=0.1 \gamma=30^{\circ}\end{array}$ & $\begin{array}{c}\text { State } 121 \\
\beta=0.1 \gamma=20^{\circ}\end{array}$ & $\begin{array}{c}\text { State } 119 \\
\beta=0.2 \gamma=30^{\circ}\end{array}$ \\
\hline & $\frac{3}{2} \rightarrow \frac{1}{2}(99 \mathrm{keV})$ & 0.90 & 1.6 & 1.5 & 6.5 \\
\hline & $\frac{5}{2} \rightarrow \frac{1}{2}(130 \mathrm{keV})$ & 1.0 & 1.6 & 1.5 & 6.5 \\
\hline & $\frac{5}{2} \rightarrow \frac{3}{2}(31 \mathrm{keV})$ & & 0.53 & 0.063 & 0.00005 \\
\hline \multirow{4}{*}{ 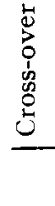 } & $\frac{3}{2} \rightarrow \frac{1}{2}(210 \mathrm{keV})$ & 2.14 & 0.05 & 0.08 & 0.00003 \\
\hline & $\frac{5}{2} \rightarrow \frac{1}{2}(240 \mathrm{keV})$ & 1.29 & 0.006 & 0.003 & 0.00003 \\
\hline & $\frac{5}{2} \rightarrow \frac{3}{2}(140 \mathrm{keV})$ & 0.11 & 0.006 & 0.36 & 1.84 \\
\hline & $\frac{5}{2} \rightarrow \frac{1}{2}(>259 \mathrm{keV})$ & & 0.002 & & 0.28 \\
\hline
\end{tabular}

The electric quadrupole transition probabilities are shown in table 7 . The asymmetric model fails to predict one of the most striking features of the observed spectrum, the large cross-over $\mathrm{E} 2$ transition probabilities involving the upper $\frac{3}{2}-, \frac{5}{2}-$ doublet. Since this is the most characteristic feature of the spectrum, it must be concluded that the simple asymmetric model does not fit the low-lying levels of $\mathrm{Pt}^{195}$. The calculations indicate that a fit of the energy spectrum alone, if only a few states are observed, can never be confirmation of the asymmetric model, since the asymmetric rotator spectrum is very rich in levels which are a sensitive function of $\gamma$, so that many different sequences of four or five levels can be reproduced.

Attempts to fit the levels of $\mathrm{Pt}^{195}$ with a symmetric rotator model seem to indicate only that the nucleus falls into the intermediate coupling region where neither the simple rotational nor the simple vibrational model can be applied. Since the particle states 119 and 121 are nearly degenerate for certain values of $\beta$ and relatively large $\gamma$, the possibility does exist that the observed level scheme can be accounted for in terms of two different particle excitations with rotational wave functions which for the $I=\frac{3}{2}-, \frac{5}{2}-$ states are strong mixtures of the two states and would therefore give rise to two $\frac{3}{2}-, \frac{5}{2}-$ doublets with strong E2 transition probabilities to the ground state. However, much more experimental information would be needed about the nucleus before such a sophisticated interpretation could be adequately tested. It should be noted, however, that no strong E2 cross-over probabilities are observed for the $\mathbf{2 +}$ levels of the neighbouring even isotopes, $\mathrm{Pt}^{194}$ and $\mathrm{Pt}^{196}$. The assumption that 
the low-lying levels of $\mathrm{Pt}^{195}$ involve two different particle excitations may therefore be reasonable.

We would like to thank Mr. R. A. Leacock for his help with the computer programs.

\section{References}

1) A. S. Davydov and G. F. Filippov, JETP (USSR) 35 (8) (1958) 303; Nuclear Physics 8 (1958) 237

2) A. Bohr, Mat. Fys. Medd. Dan. Vid. Selsk. 26, No. 14 (1952);

A. Bohr and B. R. Mottelson, Mat. Fys. Medd. Dan. Vid. Selsk. 27, No. 16 (1953)

3) C. A. Mallmann and A. K. Kerman, Nuclear Physics 16 (1960) 105;

A. S. Davydov and V. S. Rostovsky, Nuclear Physics 12 (1959) 58;

R. B. Moore, R. S. Weaver and W. White, Can. J. Phys. 37 (1959) 1036;

J. Hiura and S. Suekane, Progr. Theoret. Phys. 24 (.960) 462;

E. P. Grigoriev and M. P. Avotina, Nuclear Physics 19 (1960) 248

4) T. Tamura and T. Udagawa, Nuclear Physics 16 (1960) 460

5) T. D. Newton, Can. J. Phys. 38 (1960) 700 and Chalk River Report CRT-886 (1960)

6) S. G. Nilsson, Mat. Fys. Medd. Dan. Vid. Selsk. 29, No. 16 (1955)

7) A. S. Davydov, Proc. Int. Conf. on Nuclear ed. by D. A. Bromley znd E. Vogt (North-Holland Publ. Co., Amsterdam, 1961) p. 801

8) A. S. Davydov, JETP (USSR) 36 (9) (1959) 1103

9) B. R. Mottelson and S. G. Nilsson, Mat. Fys. Skr. Dan. Vid. Selsk. 1, No. 8 (1959)

10) G. Alaga, K. Alder, A. Bohr and B. Mottelson, Mat. Fys. Medd. Dan. Vid. Selsk. 29, No. 9 (1955)

11) K. Helmers, Nuclear Physics 20 (1960) 585

12) G. R. Satchler, Ann. Phys. 3 (1958) 273;

A. M. Lane, Rev. Mod. Phys. 32 (1960) 519;

M. H. McFarlane and J. B. French, Rev. Mod. Phys. 32 (1960) 567

13) A. K. Kerman, Mat. Fys. Medd. Dan. Vid. Selsk. 30, No. 15 (1956)

14) F. K. MicGowan and P. H. Stelson, Phys. Rev. 116 (1959) 154 